\section{KENTRON}

REVUE PLURIDISCIPLINAIR

DUMONDE ANTIQUE

\section{Kentron}

Revue pluridisciplinaire du monde antique

\section{2 | 2016}

Approches historiennes des images (I)

\title{
Rilettura ed esegesi omerica alla Scuola di Gaza : Il caso del Patroclus di Coricio
}

\section{Matteo Deroma}

\section{(2) OpenEdition}

1 Journals

\section{Edizione digitale}

URL: http://journals.openedition.org/kentron/875

DOI: 10.4000/kentron.875

ISSN: 2264-1459

\section{Editore}

Presses universitaires de Caen

\section{Edizione cartacea}

Data di pubblicazione: 31 décembre 2016

Paginazione: 151-184

ISBN: $978-2-84133-840-5$

ISSN: 0765-0590

Notizia bibliografica digitale

Matteo Deroma, «Rilettura ed esegesi omerica alla Scuola di Gaza : Il caso del Patroclus di Coricio », Kentron [En ligne], 32 | 2016, mis en ligne le 10 mai 2017, consulté le 16 novembre 2020. URL : http:// journals.openedition.org/kentron/875; DOI : https://doi.org/10.4000/kentron.875

\section{(c) (i) (9)}

Kentron is licensed under a Creative Commons Attribution-NonCommercial-NoDerivatives 3.0 International License. 


\title{
RILETTURA ED ESEGESI OMERICA ALLA SCUOLA DI GAZA : IL CASO DEL PATROCLUS DI CORICIO ${ }^{1}$
}

\author{
A mia nonna Maria, \\ $\Psi v \chi \grave{\eta} \pi \tilde{\alpha} \sigma \alpha \dot{\alpha} \theta \dot{\alpha} v \alpha \tau o \varsigma . \tau o ̀ ~ \gamma \dot{\alpha} \rho \dot{\alpha} \varepsilon ı k i v \eta \tau o v \alpha \dot{\alpha} \theta \dot{\alpha} v \alpha \tau o v$.
}

Plat., Phaedr. 245C5.

Se, negli ultimi anni, un progressivo intensificarsi di studi sulla Scuola di Gaza nel tardo-antico ${ }^{2}$ ha permesso di conoscere più approfonditamente la tecnica declamatoria di Coricio e le funzioni delle $\delta \iota \alpha \lambda \dot{\xi} \xi \varepsilon \iota \varsigma$ e $\theta \varepsilon \omega \rho i ́ a ı$ che accompagnano la sua produzione ${ }^{3}$, resta in larga misura ancora lacunoso il discorso relativo al dialogo fra il retore e i suoi modelli letterari. Infatti, al di là della raccolta dei

1. Desidero ringraziare vivamente Eugenio Amato per l'aiuto datomi nell'elaborazione di questo lavoro e per l'incoraggiamento continuo nel progetto di tesi di dottorato, in cui questo articolo si inserisce. Un ringraziamento di cuore va anche a Giampiero Scafoglio, la cui generosa disponibilità e l'approfondita conoscenza di questioni mitologiche e tradizioni omeriche hanno contribuito non poco a migliorare queste pagine. Un sentito ringraziamento anche a Paola D'Alessio e al referee anonimo per aver riletto in anteprima il contributo e averlo arricchito con alcuni preziosi consigli. Infine, un grazie ad Antonino M. Milazzo, per l'invio dell'estratto del suo Riuso mitologico e strategia retorica nel Priamo di Coricio, in Celentano et al. 2015, 297-307. La traduzione italiana dei passi greci, salvo laddove diversamente indicato, è da intendersi mia ; per quella del Patroclus, si riprende quella a cura di E. Amato e M. Deroma nel volume attualmente in corso di pubblicazione, Coricio di Gaza. Discorsi e frammenti, a cura di E. Amato, A. Corcella e G. Ventrella (= Amato et al. 2016), Leuven, Peeters (Bibliothèque de Byzantion), 2016.

2. A partire dal 2005, una serie di volumi relativi alla Scuola di Gaza hanno visto la luce. Vale la pena di segnalare almeno i principali: Saliou 2005; Bitton-Ashkelony \& Kofsky 2004; Amato et al. 2014; Amato et al. 2016. Per la rassegna bibliografica completa sugli autori gazei per il periodo 1930-2010 si rimanda ai lavori di E. Amato (Procopio ed Enea di Gaza), A. Corcella (Coricio e Zacaria) e D. Lauritzen (Giovanni di Gaza) in corso di stampa per Lustrum. Restano naturalmente validi i contributi di sintesi anteriori, fra i quali spicca Duneau 1971, 251-278. Invece, per un inquadramento più generale su Gaza nella tarda antichità, vd. Downey 1963; Glucker 1987; AA.VV. 2007; Sivan 2008, 328-347.

3. Vd., in particolare, Telesca 2011-2012, 89, n. 11, e 102-105; Lupi 2014a e Amato 2014, 3-39 (Notice introduttiva alle Dialexeis di Procopio di Gaza). 
semplici loci similes e dello studio in generale della Quellenforschung, si sente ancora oggi la mancanza di un'analisi ragionata, che si addentri nei meccanismi che regolano non soltanto la produzione del testo, ma anche la sua esecuzione ed il rapporto instaurato fra retore e pubblico, grazie all'interazione e allo scambio con le fonti letterarie ${ }^{4}$.

I pregevoli lavori di Simona Lupi ${ }^{5}$ e Aldo Corcella ${ }^{6}$ - dedicati, rispettivamente, a Erodoto, Tucidide e Demostene ${ }^{7}$ - costituiscono un primo approccio al problema. Ancora non è stato effettuato, tuttavia, un lavoro sistematico sull'auctor princeps della poesia greca, ovvero Omero. Qualche spunto di riflessione è offerto da Fotini Hadjittofi $^{8}$, che si è soffermata sul rapporto di emulazione, tendente alla competizione, instaurato da Coricio nei confronti del poeta: una competizione che si esercita nella prerogativa di organizzare e di 'dirigere', alla maniera di un maestro del coro, la materia mitica o retorica, a seconda dell'uso necessario alla riuscita

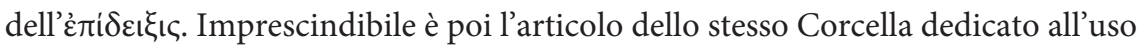
del mito a Gaza ${ }^{9}$, che ricerca i punti di sintesi e di incontro, nella città palestinese, fra cultura letteraria pagana e filosofia cristiana, partendo dall'analisi di alcuni brani coriciani e procopiani. Infine, recentissimo è il contributo di Antonino Milazzo sul riuso del mito e sulla strategia retorica nel Priamo di Coricio ${ }^{10}$.

Scopo di questo contributo è, quindi, una prima analisi ragionata del riuso e della rielaborazione di materiale omerico nel corpus coriciano, seguita da un'indagine più approfondita relativa al Patroclus (op. XXXVIII F./ R. [= decl. 10]). Questa declamazione - sul cui contenuto, struttura e tematica ho già avuto occasione di soffermarmi altrove ${ }^{11}$ - rappresenta, infatti, un caso esemplare di dialogo tra fonte omerica ed argomentazione retorica.

\section{A scuola: Omero e i Progimnasmi}

Senza volersi addentrare, in questa sede, nella ricapitolazione dei complessi rapporti e del delicato equilibrio fra eredità letteraria pagana e sviluppo dell'identità

4. Preliminari approcci in questo senso sono stati forniti da Lupi 2014a e da Telesca 2013.

5. Lupi 2009; Lupi 2010; Lupi 2014b; Lupi 2016.

6. Corcella 2005, 82-90.

7. Riprese erodotee, tucididee e finanche omeriche sfuggite a Foerster \& Richtsteig 1929 sono state di recente messe in luce e studiate da E. Amato: vd. Amato 2015; Amato 2016a; Amato 2016b e Amato 2016c.

8. Vd. Hadjittofi 2016.

9. Corcella 2013. Vd. anche Stenger 2016.

10. Milazzo 2015.

11. Vd. Deroma 2014. Per la fortuna di Coricio in età bizantina ivi compresa quella straordinaria del Patroclus, vd. Amato 2009. 
culturale cristiana all'epoca della maggiore fioritura della Scuola di Gaza, sarà tuttavia utile evocare almeno qualche indispensabile punto fermo e stabilire alcune premesse $^{12}$. Se si considerano oramai accertati il contesto profondamente cristiano in cui i retori gazei si trovarono a operare ${ }^{13}$ e il loro duplice ruolo di retori della collettività cittadina e di professori responsabili della formazione della futura élite locale, bisogna in prima istanza domandarsi perché Omero ${ }^{14}$ fosse così presente nella cultura gazea del VI secolo.

Nonostante i grandi cambiamenti prodottisi a partire dalla diffusione del Cristianesimo, il mito pagano e soprattutto i poemi omerici sono stati a Gaza e, in generale, nel Tardoantico, un elemento costitutivo dell'identità culturale, grazie ai quali il pubblico era in grado di riconoscere le proprie radici e di cui poteva rimanere - almeno in parte - un fedele seguace ${ }^{15}$. I dati "letterari" vengono ampiamente confermati dalle testimonianze iconografiche: sembra, infatti, che i personaggi e gli eventi del mito omerico fossero onnipresenti a Gaza nelle arti figurative, di cui non pochi esempi, pur non pervenuti fino a noi, sono descritti proprio dai sofisti della città palestinese nei loro discorsi ${ }^{16}$.

Poiché questi ultimi erano recitati in pubblico ${ }^{17}$, vale la pena soffermarsi su di un tipo particolare di discorso e di epideixis, la declamazione, la declamazione, che da esercizio prettamente scolastico, divenne presto discorso di intrattenimento in

12. Si rimanda alla bibliografia richiamata alla n. 2. Sull'importanza della commistione di elementi culturali pagani e cristiani si aggiungano: Amato 2010a; Amato 2010b; Amato 2012; Amato 2014, 3-39; Ventrella 2011; Ventrella 2011-2012; Ventrella 2012.

13. I dubbi sulla effettiva aderenza al cristianesimo di Coricio e del suo maestro Procopio sono sostanzialmente messi a tacere da Fozio (Phot., Bibl., cod. 160, 102b [II, p. 122 Henry]), il quale tuttavia si stupiva del frequente inserimento nei loro scritti di miti e storielle pagane, perfino mescolati ai soggetti sacri. Sul codice foziano in generale, si veda l'attenta analisi di Amato 2009, 270-274 e 287-289.

14. Quando, in questa sede, si parlerà di mito, si intenderà soprattutto quello Omerico, con riferimento però non soltanto all'Iliade e all'Odissea, ma anche ai poemi del Ciclo.

15. Coricio - come il suo maestro Procopio - mescola volentieri elementi di ispirazione cristiana con reminiscenze mitologiche. Cfr., tra l'altro, l'attenta analisi di Stenger 2016; su Procopio di Gaza, cfr. Amato 2014, XI-LXXXV (Introduction générale), e 3-39.

16. Di notevole interesse per la conoscenza dello straordinario patrimonio artistico della città nel tardo-antico sono, per esempio, le due Descrizioni procopiane, l'una dedicata all'inaugurazione di un monumentale orologio meccanico ad acqua (op. VIII Amato), composto da figure mobili riguardanti il mito di Eracle e l'altra (op. IX Amato) relativa ad un grande affresco su soggetti del ciclo omerico e tragico. Sulla ricchezza di questi testi, vd. la Notice introduttiva alle due opere in Amato 2014, 117-220 e L. Thévenet, «L'Ekphrasis eikonos de Procope de Gaza: visite guidée d'une tragédie», in Amato et al. 2016.

17. Nella vasta bibliografia relativa alla recitazione in pubblico dei discorsi dei retori gazei, oltre a Amato et al. 2014, vd. anche Renaut 2005 e Amato 2014, 117-220. 
occasione di manifestazioni pubbliche e feste ben precise ${ }^{18}$. Una delle caratteristiche di questo tipo di esercizio è l'importante e ben individuabile ricorso a esempi e a storie mitologiche: soggetti privilegiati sono, in effetti, le vicende di eroi ed eroine dell'epos, cui i retori attribuiscono la parola per esprimere idee e trasmettere messaggi ${ }^{19}$.

A scuola, poi, tra gli esercizi progimnasmatici ${ }^{20}$ di livello più avanzato su cui erano chiamati a cimentarsi gli allievi, un posto di rilievo spettava anche alla riscrittura - sotto forma di parafrasi o metafrasi - di testi poetici ${ }^{21}$; ma siffatte riscritture erano anche veri e propri momenti di prova del retore - a lezione, come davanti ad un pubblico più ampio -, poiché egli si trovava quasi a competere con il modello - omerico o poetico in generale -, impegnandosi ad impressionare il più possibile l'uditorio e a veicolare insegnamenti ai propri allievi ${ }^{22}$.

18. Cfr. Pernot 2000, 204: «Quand un déclamateur en vue donnait une séance, dans une école ou dans un lieu public de la cité (odéon, salle du Conseil, théâtre), l'affluence était grande et le public comprenait, outre les étudiants et les collègues, des personnages de qualité extérieurs au monde de l'école»; cfr. anche ibid., 200-207 e 271-272. Questa evoluzione deve essere inquadrata nell'importante dimensione pubblica di intrattenimento in cui il ruolo del retore si trova assimilato a quello di

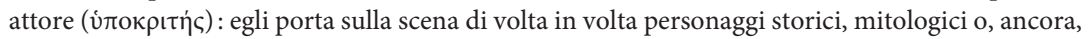

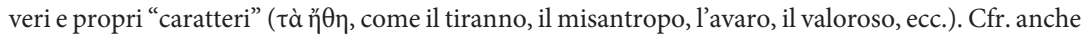
Civiletti 2002a, 62-69; Civiletti 2002b, 46; Russell 1983, 74-86, e Amato 2016c.

19. Particolarità della pratica retorica greca di età imperiale è la paziente ricerca svolta sulle fonti storiche e mitologiche per ricostruire uno scenario che sembri reale, in cui inserire una sottile, ma solida argomentazione. A questo proposito, vd. Amato \& Sauterel 2010 e relativa bibliografia.

20. Per una rapida quanto esaustiva indagine di tutto il repertorio di questi esercizi preparatori si rimanda a Patillon 1990, 141-155 e alla relativa bibliografia. Nel Patroclus, per citarne solo alcuni,

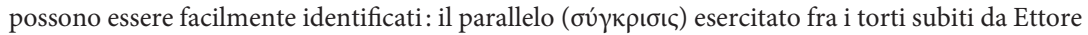

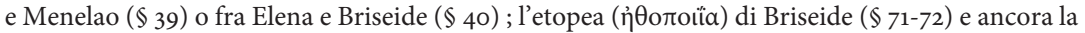
descrizione ( $(\check{\kappa} \kappa \rho a \sigma \iota)$ ) dell'opera d'arte (\$69); non vanno dimenticati nemmeno tutte le sentenze

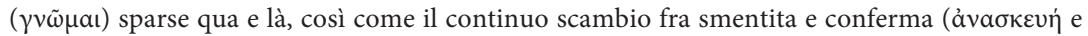

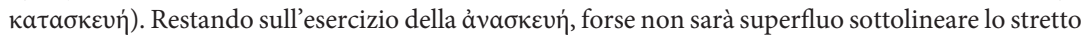
rapporto esistente fra le dinamiche dell'insegnamento scolastico e molte delle declamazioni del retore gazeo. Alcune di queste, tra cui il Patroclus, potrebbero anzi essere interpretate proprio come ritrattazioni generali - delle refutationes, appunto - della vulgata nel loro rivaleggiare con il modello poetico. Coricio poteva disporre, in tal caso, del noto esempio offerto dal Troiano di

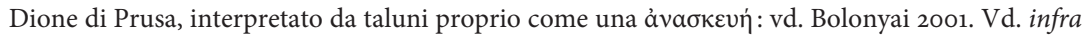
e Conclusioni.

21. Numerose sono le testimonianze, a partire dall'età ellenistica, di papiri contenenti brevi esercizi ad opera di studenti che ricopiano il testo omerico non in maniera pedissequa, bensì volgendolo in prosa e sostituendo le parole più difficili con altre della koiné. Cfr., in particolare, gli studi di Cribiore 2009; Cribiore 2001a. Sulla massiccia presenza di Omero, cfr. sempre ad opera di Cribiore 1993; Cribiore 1994, 4-6; Cribiore 1996; Cribiore 2001b, 194-210; 220-230.

22. Bisogna tenere presente che, in ambito gazeo, imprescindibile era probabilmente la parafrasi allestita da Procopio, maestro di Coricio, cui fa riferimento anche Fozio (Phot., Bibl. cod. 160 [II, p. 122,

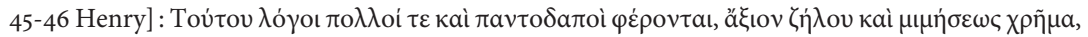

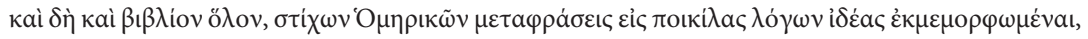

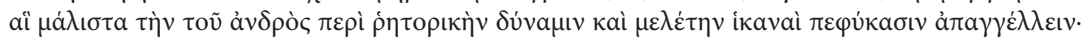


Nel periodo tardoantico, poi, la parafrasi e la metafrasi assursero al ruolo di genere letterario vero e proprio, sviluppandosi in forme sempre più complesse e varie anche per l'esigenza dei prosatori cristiani, che si trovarono a parafrasare testi biblici per finalità critiche ed esegetiche ${ }^{23}$.

Tuttavia, già Teone aveva segnalato quanto fosse rischioso affrontare declamazioni imbastite su soggetti mitologici ${ }^{24}:$ il retore non doveva assolutamente cadere in un discorso menzognero, errato, impossibile o non plausibile (åri $\theta a v o v)^{25}$.

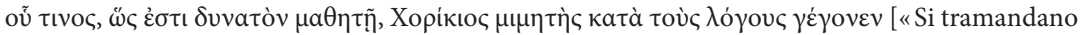
di Procopio molti scritti e di differente genere, un'opera degna di suscitare invidia e imitazione, in particolare un intero libro, in cui parafrasa i versi di Omero in una grande varietà di forme: si tratta dell'opera più adatta a far risaltare l'abilità retorica dell'autore. Ė questo tale che Coricio, per quanto è possibile a un allievo, imitò nei propri discorsi» [trad. Amato in Fozio, Biblioteca, N. Bianchi, C. Schiano (eds), Pisa, Edizioni della Normale, 2016].). A questo proposito, recentemente, Amato 2014, XLIII-XLV e, in particolare, n. 129, ha ipotizzato che le Metafrasi di versi omerici (F VI/1-2 Amato) di Procopio avessero un contenuto più retorico-scolastico che sofistico e che esse fossero impiegate dal maestro a lezione. La tradizione dell'esercizio di metafrasi è antica, oltre che molto varia: Amato ipotizza che Procopio potrebbe aver avuto a disposizione le Metapoieseis del retore Sopatro, una monografia dedicata alla parafrasi per mostrare ai propri allievi la varietà di questo tipo di esercizio. Amato, inoltre, si chiede se il Sopatro in questione non possa essere identificato con il sofista di V secolo di cui fa menzione Zacaria Scolastico nella Vita di Severo (p. 12, 2-3 Kugener); in tal caso, il retore potrebbe essere stato in contatto anche con Procopio. Sopatro, afferente al circolo di Horapollon, ebbe tra i suoi discepoli Severo di Antiochia, lo stesso Zaccaria e fu forse in relazione con Enea di Gaza (l'ep. 9 Massa Positano ha per destinatario un Sopatro) : l'ipotesi accattivante cui giunge Amato è, dunque, che sarebbe davanti a questo maestro rinomato che Procopio avrebbe vinto, in gioventù, un concorso poetico-retorico ad Alessandria, come attesta Coricio nell'Epitafio del suo maestro (vd. Choric., op. VIII, 15, p. 114, 23-115, 3 F. / R.).

23. Nacquero anche nuove forme di libri e di lettura, legate a una nuova fascia di fruitori del testo scritto: proprio il testo omerico diviene dunque oggetto non solo di parafrasi, ma anche di trasposizione figurata; cfr. Cavallo 2001. Caso sicuramente emblematico è l'esemplare manoscritto noto come Iliade ambrosiana (F 205 inf.). Nel testimone manoscritto ambrosiano, come in altri consimili, trovano uno spazio privilegiato i passaggi riguardanti Achille: le sue vicende, precedenti e successive alla guerra di Troia, sono raccontate in modo vario anche in altri codici, per l'intera durata del periodo bizantino.

24. Theon, Prog. 76.8-11, p. 35 Patillon: «[...] parce que le fabuliste reconnaît lui-même que ses compo-

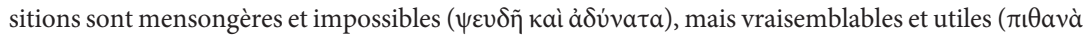
$\delta \grave{\varepsilon}$ кaì $\dot{\omega} \varphi \dot{\varepsilon} \lambda(\mu \alpha)$, la contestation montrera que ses propos sont invraisemblables et inopportuns

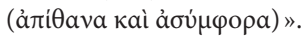

25. Theon, Prog. 76.34-77.9, p. 36-37 Patillon «Il y a invraisemblance lorsque les faits ou les paroles sont possibles, mais non crédibles, soit en raison de la personne à qui on attribue le fait ou la parole, soit en raison du lieu où on situe tel fait ou telle parole, soit en raison du temps où l'on place tel fait ou telle parole, soit en raison de la manière du fait ou de la parole, soit en raison de la cause de ces mêmes faits ou paroles, lorsque nous disons qu'il n'est pas plausible que telle personne ait fait ou dit telle chose en tel lieu, en tel temps, de telle manière ou pour telle cause». Anche secondo Ermogene i discorsi che permettono l'inserimento delle categorie dell'inverosimile, dell'impossibile e delle contraddizioni sono necessariamente fallaci. Cfr. Patillon 1997a, 113; 310; 
Questa contaminazione fra $\lambda$ ó $\gamma$ oc e $\mu \tilde{v} \theta$ oc è una pratica antica, ma ebbe uno sviluppo considerevole a partire dall'epoca della Seconda Sofistica, per poi raggiungere il massimo incremento proprio con retori quali Libanio ${ }^{26} \mathrm{e}$ i Gazei. Come ha recentemente osservato Milazzo in merito al Priamo di Coricio ${ }^{27}$, il "passato mitologico" viene sempre più razionalizzato nel Tardoantico, manipolando la tradizione epica nota e sovvertendo, quindi, alcune delle aspettative del pubblico. Questa razionalizzazione, tuttavia, non va intesa come una opposizione totale, ma come una vera e propria riscrittura, che è specchio della complessa varietà mitica e che risponde a un duplice sistema di attese del pubblico: da un lato, l'ammirazione per la potenza della retorica sul piano artistico e didattico, grazie al dispiegamento di elaborati modelli di persuasione; dall'altro, il confronto con il Poeta per eccellenza ${ }^{28}$. Importante è, tuttavia, il ricorso alle categorie del "verosimile" e del "possibile", per integrare la "verità omerica" con un discorso epidittico volto a persuadere l'uditorio.

Un'analisi di questo genere, applicata anche al Patroclus e alle altre declamazioni mythologicae coriciane, permetterebbe dunque di provare a chiarire meglio il contesto

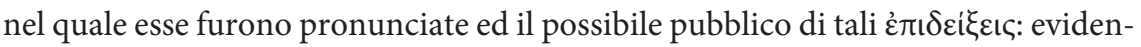
temente, quanto più un testo tradisce un massiccio impiego di citazioni poetiche, tanto più il suo pubblico e i suoi destinatari si prospettano essere gli accorti allievi del retore $\mathrm{o}$, in ogni caso, un pubblico relativamente selezionato ${ }^{29}$.

313; 490. Cfr. Arist., Po. 1460a26-27, sul criterio del possibile e della verosimiglianza (

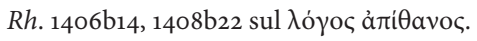

26. Se si considerano i Progymnasmata del sofista antiocheno (Libanii opera, VIII Foerster), è possibile riscontrare che si tratta di una antologia di esercizi svolti e corretti, fra i quali figurano molti contenuti da ricondurre ad argomenti di epica e delle tragedie classiche. Mi limito, a questo proposito, a ricordare solo Lib., Narr. 1; 5-7; 14-15; 17-18; 23; 27; 31; Ref. 1; 2; Conf. 1-3; Enc. 1-4; Vit. 1-2; 7 ; Comp. 1-2; Eth. 1-17; 21-25; Descr. 13-23; 28.

27. Milazzo 2015, 297-299.

28. Quando qui si parla di pubblico bisogna tenere ben presente che restano aperte varie possibilità : esso può essere reale, composto di studenti o di indistinti ascoltatori, oppure meramente fittizio. Qualora si presupponga un pubblico effettivamente reale - e di questo aspetto non vi è motivo di dubitare - il cui livello culturale è assolutamente disomogeneo e varia sensibilmente. La declamazione, come avremo modo di chiarire in seguito, si presta perciò a diversi livelli di analisi e lettura.

29. Per quanto fosse possibile che il pubblico stesso, talvolta, proponesse il soggetto argomentativo da sviluppare, in generale, tuttavia, si tratta di un'occasione particolare, che offre lo spunto al retore per lo sviluppo di una determinata tematica o di un mito: ad esempio, un episodio mitologico studiato in classe con gli studenti poteva diventare la materia sulla quale imbastire un discorso sull'amicizia o creare i presupposti per istituire paragoni con personaggi della realtà locale - attuale o storica. Per quanto riguarda questo ipotetico pubblico valga quanto detto supra p. 153, n. 17. Una rilevanza

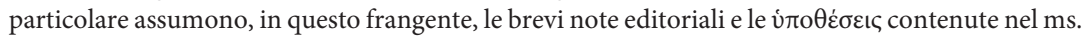
Madrid 4641 (N-101), sulla cui attendibilità si è però dubitato. 
È ben noto che, per tutta l'età imperiale e durante la tarda antichità, in ambito scolastico dominava un certo conservatorismo: ai fini dell'insegnamento, si privilegiarono i grandi autori antichi (Omero - con una preferenza per l'Iliade rispetto all'Odissea -, Erodoto, Tucidide, Euripide, Platone, Demostene, Menandro, ecc.) rispetto a quelli più recenti ${ }^{30}$.

Sebbene Coricio precisi di non aver mai scritto opere di poesia ${ }^{31}$, nondimeno, nel Patroclus e nelle altre due declamazioni mitologiche egli dimostra di essere un fine conoscitore dei poemi omerici, con una propensione per alcuni canti in particolare $^{32}$ : a proposito di tali testi, egli fa sfoggio di una competenza analitica notevole, sintomatica di un intenso studio svolto con i propri studenti. Omero è, dunque, non solo citato, ma anche commentato e offre lo spunto per l'elaborazione retorica di alcuni passaggi delle $\theta \varepsilon \omega$ piaı e, naturalmente, delle tre declamazioni mitologiche.

Ma la citazione omerica è - innanzitutto - garanzia di realtà storica e, dunque, nella maggior parte dei casi, di verità. Una distinzione che però neppure Stenger ha affrontato è quella fra opere pensate per essere declamate di fronte a un pubblico riunito per una celebrazione ufficiale cittadina e altre, pensate e prodotte per una cerchia più ristretta, che vuole trarre dalla $\dot{\varepsilon} \pi i \delta \varepsilon \xi \xi ı \varsigma$ un diletto e un insegnamento retorico ${ }^{33}$.

30. Sul sistema di istruzione nella tarda antichità cfr. Petit 1957, 84-85; Booth 1979; Kaster 1983; Marrou 1994, 243 sqq. ; Browning 2001, 862. Alla Scuola di Gaza poi, come ha notato Duneau 1971, 251-257, la collaborazione fra insegnanti di primo e secondo grado era fondamentale: i retori erano aiutati da veri e propri assistenti - i $ү \rho \alpha \mu \mu \alpha \tau$ «ó,, o insegnanti di primo grado -, che si occupavano del gran numero di studenti sotto la loro tutela.

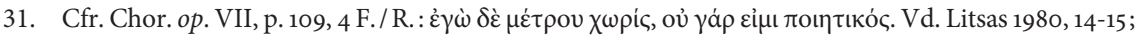
20-24. Può anche darsi che in questa affermazione coriciana possa leggersi una critica piuttosto comune in ambito retorico, ovverosia che lo stile poetico fosse generalmente da evitare. L'espressione più famosa di questo concetto si trova in Theon, Prog. 71, 7-10, p. 16 Patillon ( «Il faut veiller aussi à l'arrangement des mots et à enseigner tout ce qui permet d'éviter un arrangement vicieux, en particulier l'expression métrique et rythmée» [trad. Patillon]) e 81, 8-11, p. 42 Patillon («En ce qui concerne les mots, celui qui recherche la clarté doit éviter l'emploi des mots poétiques, forgés, métaphoriques, archaïques, étrangers et homonymes. Sont poétiques tous ceux qui nécessitent une interprétation»). Come nota lo stesso Patillon (cfr. Patillon 1997b, 131, n. 108), "Aristote déjà met en garde contre l'emploi en prose d'une expression trop poétique (Rhétorique 3, 8, 1408b21-24)».

32. Sulla predilezione di Coricio per alcuni canti omerici vd. nel dettaglio i risultati di C. Telesca, «Erudizione e realtà sociale negli Epitalami di Coricio di Gaza», in Amato et al. 2016, p. 56 n. 9. Invece, sulla presenza di Omero in epoca tardoantica e bizantina cfr. soprattutto Hadjittofi 2016 e gli studi di Browning 1975 e Browning 1992.

33. Quando Stenger 2016, 16, infatti, riferisce del bisogno di Coricio di avvisare il pubblico che certe citazioni provengono dalla letteratura antica e non sono, talvolta, degne di fede, manca di precisare che questa sorta di diffidenza verso la letteratura pagana è più presente giocoforza in un contesto ufficiale, in cui le convenzioni culturali cristiane impongono il ricorso più rigido a un'accurata selezione del materiale. 


\section{Il caso del Patroclus}

Il Patroclus coriciano si inserisce perfettamente in questo contesto e nelle peculiari dinamiche che lo caratterizzano: è un discorso fittizio, composto per una recitazione orale di fronte ad un pubblico eterogeneo; un discorso in cui il declamatore, assunta la maschera di un personaggio - in questo caso, mitologico - si dimostra, intenzionato a raggiungere un obiettivo specifico: persuadere un interlocutore - anch'esso un personaggio mitologico - a cambiare avviso ${ }^{34}$.

Come abbiamo già avuto modo di accennare in altra sede, tutte e tre le declamazioni a soggetto mitologico presenti nel corpus di Coricio gravitano attorno alla leggenda della guerra di Troia ${ }^{35}$.

Achille, in questo caso, è il destinatario di una appassionata arringa dell'amico suo più caro, costretto oramai alla supplica, non avendo trovato, fino ad allora, alcun modo per farlo desistere dal suo proposito. La tecnica di persuasione che Patroclo ha adottato nell'Iliade, cioè il pianto per muovere l'animo di Achille, non ha sortito alcun effetto: Coricio immagina, di conseguenza, il discorso che il giovane avrebbe dovuto (e potuto) pronunciare per riuscire nel suo intento, ovvero un elogio della virtù guerriera dell'amico e, allo stesso tempo, un drammatico ritratto della situazione in cui versa lo schieramento acheo.

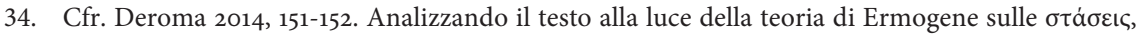
ovvero gli status o "posizioni" che, per l'appunto, l'oratore deve occupare in un certo dibattimento,

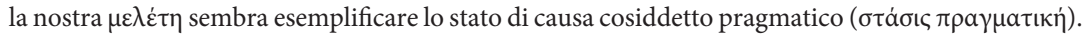
A questa conclusione si può arrivare seguendo il percorso indicato dal trattato ermogeniano: la scelta di Achille di abbandonare a se stesso lo schieramento acheo, così come le sue conseguenze, sono evidenti a tutti; dunque, si può parlare proprio di azione completa ed evidente; la qualità, poi, di tale azione, fra i tredici possibili status, è definibile come pragmatica, proprio perché i risvolti di questo caso e la sua possibile soluzione riguardano esclusivamente il futuro, al fine di cambiare quella che è la situazione attuale. Il discorso, perciò, sarà volto a concludere il processo di convincimento di Achille, facendo leva, naturalmente, sulle astuzie retoriche che la trattatistica prevedeva per questo genere di performance.

35. Per una breve analisi del contenuto della declamazione, cfr. Deroma 2014, 151-166. Nel Polidamante e nel Priamo, i Troiani assediati si riuniscono in assemblea per negoziare la proposta di Achille di schierarsi al loro fianco in cambio del matrimonio con Polissena. Le due pièces, come giustamente notato da Schouler 2005, 123-125, sono costruite in forma di antilogia. Esse non si concentrano soltanto sulla figura di Polissena, ma delineano entrambe - seppur non in maniera diretta - il carattere di Achille. Secondo il saggio consigliere dei Troiani, il figlio di Peleo, del quale egli sottolinea tutte le virtù militari e la travolgente passione amorosa, potrebbe risollevare le sorti del conflitto a favore del popolo assediato, mettendo fine alla disastrosa guerra; la risposta dell'anziano re di Troia, invece, si concentra quasi esclusivamente sui suoi difetti caratteriali quali l'insubordinazione, l'eccessiva passionalità e soprattutto l'ira selvaggia, sconfinata nell'oltraggio al corpo di Ettore. L'etica della moderazione dell'uno a favore della collettività, contro l'orgoglio di un padre. Anche nel Patroclus, naturalmente, Achille è in qualche modo protagonista per il tramite delle obiezioni che l'autore immagina egli sollevi al suo discorso e per il modo nel quale l'oratore ne delinea le caratteristiche. 
Rifacendosi direttamente ad alcuni discorsi che compongono la materia iliadica, il retore vuole mostrare al proprio pubblico per quale motivo tutti i compagni - Patroclo incluso - che avevano cercato di smuovere Achille avessero fallito. Quello che Coricio presenta all'uditorio è un discorso di rielaborazione e di commento al testo iliadico, con cui egli instaura un dialogo serrato.

Come già osservato ${ }^{36}$, doveva certamente rappresentare una forte tentazione per qualsivoglia retore immaginare di imbastire un grande discorso per l'eroe Patroclo, che nella scena dell'ambasceria a Achille da parte di Ulisse, Fenice e Aiace, si ritrova a impersonare il ruolo di un "majordome efficace et discret ${ }^{37}$. Del resto, variazioni sul tema dell'ambasceria erano già state sperimentate da Elio Aristide (Legatio ad Achillem, or. XVI Keil), ma soprattutto da Libanio (Achillis ad Vlixem antilogia, decl. 5 Foerster) e - come appena ricordato - dal maestro di Coricio stesso, Procopio di Gaza, nella quarta Etopea intitolata Fenice (op. VII Amato).

36. Schouler 2005, 121-122.

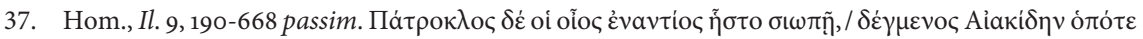

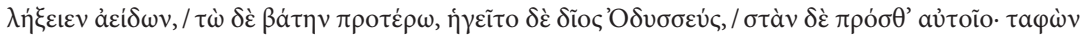

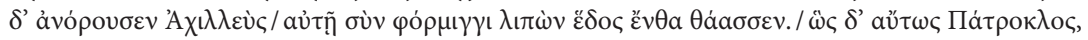

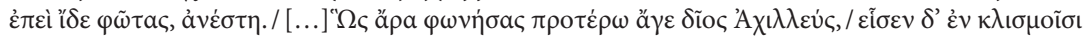

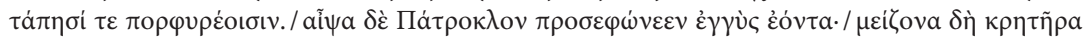

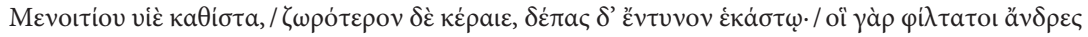

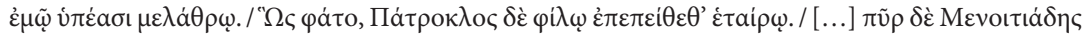

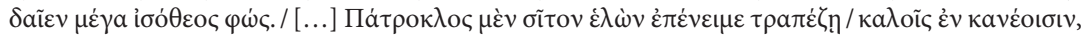

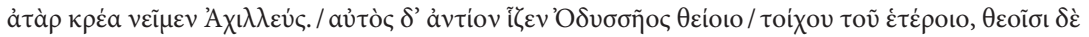

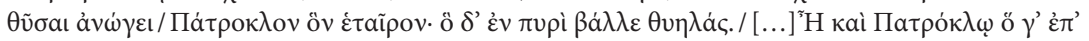

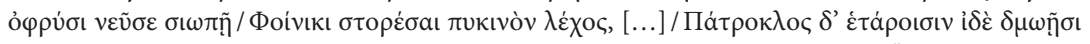

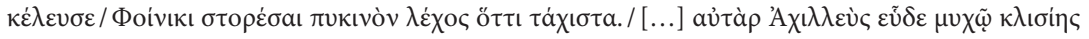

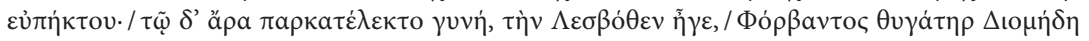

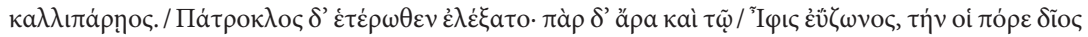
'A $\ \backslash \lambda \lambda \varepsilon \dot{v} \varsigma$. ( di fronte a lui sedeva in silenzio Patroclo, solo, e attendeva che il discendente di Eaco ponesse fine al suo canto. Si fecero avanti i messi, li guidava il glorioso Odisseo, e davanti a lui si fermarono; stupito Achille si alzò dal suo seggio, tenendo in mano la cetra; si alzò anche Patroclo quando vide gli eroi. [...] Disse così e li fece entrare Achille glorioso, li fece sedere su seggi e tappeti di porpora, e a Patroclo disse, che gli era vicino: «Prendi un orcio più grande, figlio di Menezio, mescola più vino e dà una coppa a ciascuno; gli amici più cari sono oggi sotto il mio tetto ». Disse, e Patroclo obbedì all'amico. [...] Accese un gran fuoco il figlio di Menezio, l'eroe simile a un dio. [...] Arrostita la carne, la depose nei piatti Patroclo, poi prese il pane e lo distribuì sulla tavola entro canestri bellissimi mentre Achille distribuiva la carne; egli stesso sedette poi davanti al divino Odisseo, sul lato opposto, e invitò il suo compagno a sacrificare agli dei; Patroclo gettò le offerte nel fuoco; e tutti tesero allora le mani verso i cibi pronti e imbanditi. [...] Disse, e in silenzio fece cenno a Patroclo di preparare a Fenice un morbido letto [...]. Ai compagni e alle schiave Patroclo intanto ordinava di preparare al più presto a Fenice un morbido letto ; [... Achille invece dormiva in fondo alla tenda ben costruita e accanto a lui giaceva una donna di Lesbo, la figlia di Forbante, la bella Diomeda; da un'altra parte si distese Patroclo e accanto a lui era la snella Ifi che gli donò Achille glorioso quando conquistò la rocca di Enieo, l'alta città di Sciro». Le traduzioni di tutti i successivi passi iliadici sono quelle di M. G. Ciani, in Ciani \& Avezzù 1998. 
La grande novità coriciana è, senza dubbio, l'aver dato voce - per quello che ci è dato sapere - per la prima volta a Patroclo. L'eroe che domina declamazioni mitologiche ed esercizi progimnasmatici è, difatti, normalmente Achille ${ }^{38}$ : i suoi sentimenti opposti di ira e amore, il suo disprezzo per la sorte dei compagni e la decisione tardiva di riprendere le armi dopo la morte dell'amico Patroclo, erano state scandagliate in più modi dai retori ${ }^{39}$.

Quello che, tuttavia, è passato finora inosservato, è che il Patroclus non intende tanto inserirsi sull'ambasceria del canto IX $^{40}$ - in cui il Meneziade non parla -, ma piuttosto sul canto XVI, l'unico vero tentativo di Patroclo di argomentare il suo dissenso verso l'amico e, in definitiva, il discorso di addio fra i due.

Se si passano ad analizzare la $\theta \varepsilon \omega$ pia $^{41}$ premessa alla declamazione e l'incipit della tirata di Patroclo ${ }^{42}$, si nota come Coricio innesti l'attacco del suo discorso proprio criticando l'atteggiamento tenuto da Patroclo nei primi versi del canto XVI:

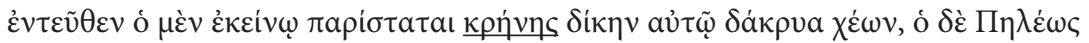

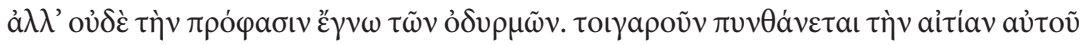

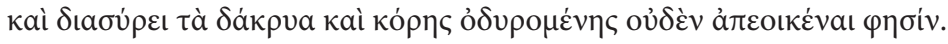

«Ecco perché, mentre l'uno [i. e. Patroclo] sta accanto a quello, versando lacrime per lui come una fontana, il figlio di Peleo neppure capisce il motivo dei lamenti. Perciò gliene domanda la causa, si fa beffa delle lacrime e dice che non è per nulla diverso da una fanciulla che piange ${ }^{43}$.

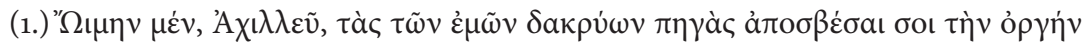

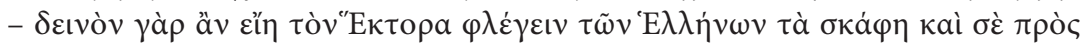

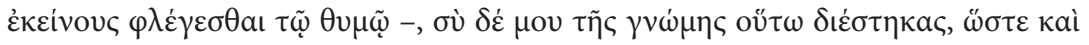

38. Cfr. n. 26.

39. La caratterizzazione di Achille nella retorica progimnasmatica e, naturalmente, nelle tre declamazioni mitologiche coriciane, costituirà un capitolo della mia tesi di dottorato. Uno studio similare, naturalmente, sarà riservato a Patroclo. Tale analisi può ora beneficiare del recentissimo articolo di Schouler 2015, dedicato alle diverse sfaccettature del personaggio iliadico, esemplificate da Libanio.

40. Il IX canto iliadico, quello retorico per eccellenza, è ripreso da Coricio solo per alcuni motivi: innanzitutto gli consente di ripercorrere, come vedremo, le ragioni del fallimento dei tre ambasciatori, ma soprattutto di innestare una vera ed efficace nuova ambasceria, che idealmente sostituisce la prima.

41. La theoria costituisce una "prefazione" programmatica, nella quale il retore spiega al proprio pubblico le motivazioni personali che lo spingeranno a trattare l'argomento nella maniera in cui il pubblico sta per ascoltarla. Inoltre, questa "osservazione teorica" o "esposizione introduttiva" serve a dare sfoggio della cultura letteraria dell'autore e della sua competenza nella gestione delle tecniche retoriche.

42. All'inizio della declamazione, a tale ripresa indiretta del discorso di Achille, sarà data la possibilità a Patroclo di rispondere, riprendendo direttamente - come vedremo di seguito - la sequenza iliadica.

43. Chor., op. XXXVIII (= decl. 10), p. 435, 2-5 F./R. 


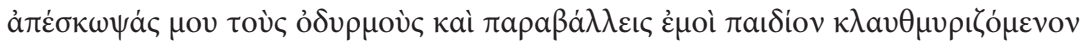

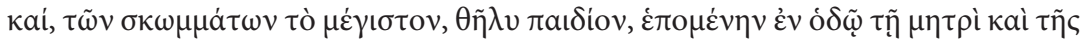

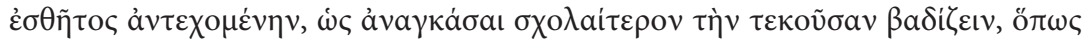

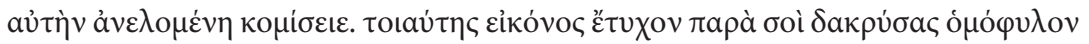

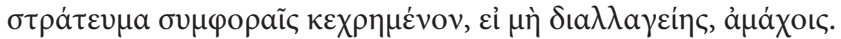

1. «Credevo, Achille, che lo scorrere a rivoli delle mie lacrime spegnesse la tua ira - sarebbe, infatti, terribile che Ettore incendiasse le navi dei Greci e tu incendiassi in cuor tuo contro costoro - ma tu ti sei a tal punto distaccato dal mio pensiero da deridere i miei lamenti e da paragonarmi ad un bimbo piagnucoloso e, insulto peggiore di tutti, ad una bambinetta, che rincorre per strada la madre e ne afferra la veste, così da costringere la genitrice a camminare più lentamente, in modo che la porti in braccio. Tale è l'immagine che hai di me per il fatto che ho pianto un fraterno esercito che ha affrontato sciagure, insuperabili se tu non fossi riconciliato ${ }^{44}$.

Confrontiamolo ora con Hom., Il. 16, 2-11, ovvero con il discorso di Achille indirizzato a Patroclo nel quale l'eroe, dopo aver visto piangere l'amico, lo paragona effettivamente a una bambinetta :

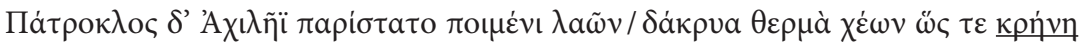

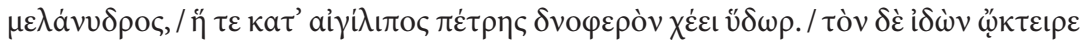

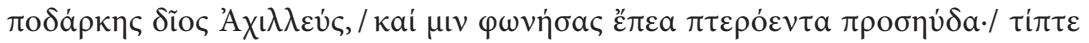

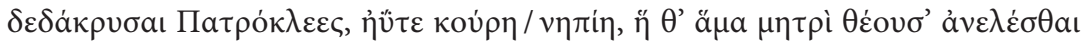

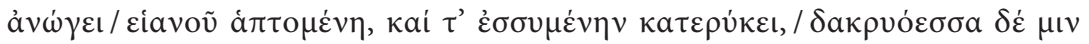

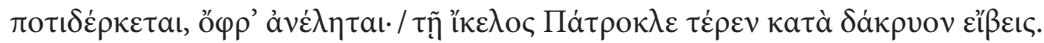
«E davanti ad Achille, condottiero di eserciti, Patroclo apparve piangendo : pareva una fonte di acqua bruna che versa fiotti scuri da un alto dirupo ${ }^{45}$. Lo vide e ne provò pietà il divino Achille dai piedi veloci, e si rivolse a lui con queste parole: 'Perché piangi, Patroclo, come una bimba piccina che corre accanto alla madre e chiede di essere presa in braccio, si attacca alla sua veste e le impedisce il cammino, e piange guardandola di sotto in su, per essere sollevata da terra; come una bimba tu piangi, Patroclo'» ${ }^{46}$.

Nella $\theta \varepsilon \omega \rho i a$, il retore riprende ambedue i termini di paragone omerici, commentandoli più ampiamente e dimostrando di averne capito il diverso significato.

44. Chor., op. XXXVIII (= decl. 10), p. 437, 7-438, 3 F. / R.

45. Il pianto del figlio di Menezio è paragonato ad una sorgente di acqua scura che sgorga da una roccia scoscesa: quasi gli stessi moduli espressivi erano stati utilizzati per indicare le lacrime di

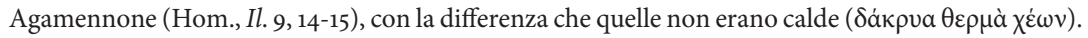
Lo saranno, invece, quelle di Achille, una volta visto il corpo dell'amico, disteso sul feretro (Hom., Il. 18, 235). Sul pianto di Agamennone, che Coricio riprende dal suddetto passaggio iliadico, cfr. $\$ 13$.

46. Hom., Il. 16, 2-11. 
Nell'Iliade, infatti, Omero presenta il pianto dell'eroe prima tramite una descrizione oggettiva che richiama un elemento naturale, ossia la fonte ( $\underline{\rho \eta \eta} v \eta$, ripreso in entrambi i testi); successivamente, introduce un paragone soggettivo, relativo alla percezione che di quel pianto ha avuto Achille (la bambina). Coricio, a differenza di Omero, segnala che il Pelìde non ha capito la ragione di tale pianto e spiega, così, per quale motivo arrivi a irriderlo paragonandolo ad una femminuccia.

Il sofista conclude poi la sua osservazione teorica anticipando quelle che saranno le strategie messe in opera dal suo personaggio, in aperta opposizione con quelle dell'eroe iliadico. Quest'ultimo, infatti, nel suo accorato quanto lacrimevole discorso, non aveva fatto altro che seguire il consiglio di Nestore ${ }^{47}$ : il re di Pilo lo aveva esortato a convincere Achille a permettere almeno a lui, suo valoroso amico, di scendere in battaglia. Tornato alla tenda, Patroclo spinge indirettamente Achille a tornare in battaglia, evocando le difficoltà e le sofferenze degli Achei, ma non gli chiede esplicitamente di intervenire. In definitiva, egli cerca piuttosto di convincerlo, tra le lacrime, a lasciarlo partire al posto suo ${ }^{48}$.

Allora, come abbiamo visto, nell'iniziare la declamazione, il Patroclo coriciano risponde alla critica che l'amico gli ha rivolto, di essere simile a una fanciulla piagnucolosa; ma il discorso - che nell'Iliade è costituito dalla manciata di versi che decretano il fallimento della retorica delle lacrime e, soprattutto, conducono Patroclo alla morte - si trasforma nella lunga tirata di $75 \$ \$ \$$. Riportiamo, anche questa volta, entrambi i passi :

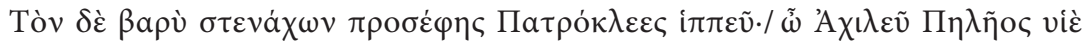

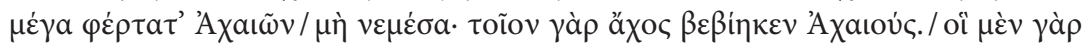

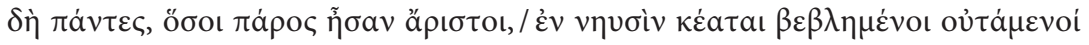

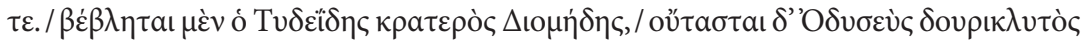

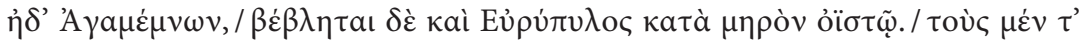
ỉn

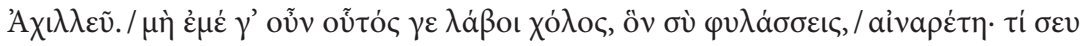

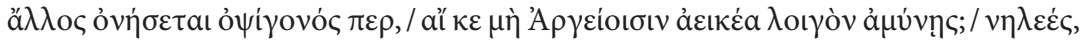

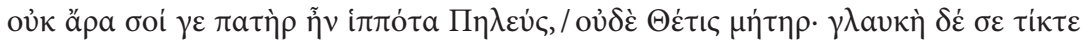

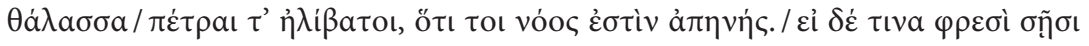

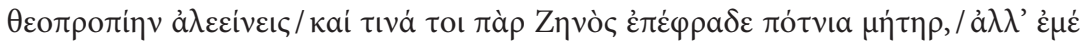

47. Cfr. il discorso di Nestore a Patroclo in Hom., Il. 11, 656-803.

48. Va in questa sede segnalato un altro richiamo sotteso - nella $\theta \varepsilon \omega \rho i \alpha$ - al discorso iliadico di Patroclo: agli amici è concesso esprimersi senza timore e, difatti, nell'Iliade, il figlio di Menezio è l'unico che potrebbe azzardarsi a criticare in maniera tanto schietta e vigorosa Achille (Chor., $o p$.

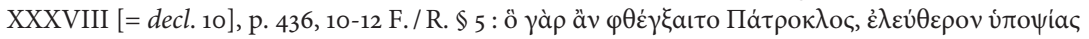

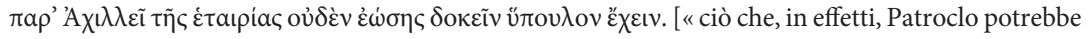
dire libero da timore dinanzi ad Achille, dal momento che la loro intima amicizia non sembra avere nulla di ingannevole»]). 


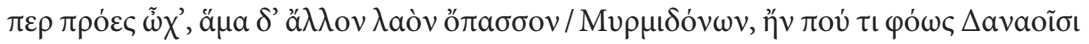

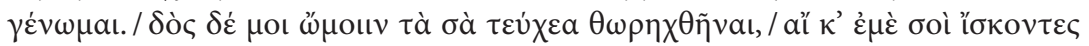

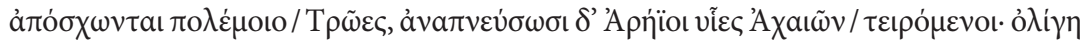

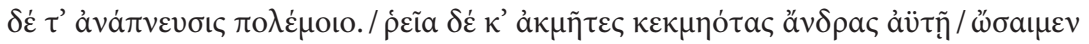

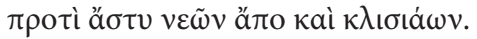

«Tra le lacrime così gli rispondesti, Patroclo, guidatore di carri: 'Achille, figlio di Peleo, che degli Achei sei il più forte, non adirarti: grande è il dolore che ha colpito gli Achei. Quelli che erano prima i più forti, giacciono sulle navi, feriti di freccia o di lancia. Colpito è il figlio di Tideo, il forte Diomede, feriti Odisseo dalla lancia gloriosa e Agamennone; una freccia ha raggiunto alla coscia anche Euripilo. Si affannano i medici, esperti di farmaci, per curare le loro ferite. E tu rimani inflessibile, Achille. Mai debba prendermi un'ira come quella che serbi dentro di te, tremendo guerriero : chi mai trarrà vantaggio da te in futuro, se non allontani dagli Achei il disastro tremendo? Uomo spietato, certo non ti fu padre il guidatore di carri Peleo, e Teti non fu tua madre; le acque azzurre del mare ti hanno generato e le rocce scoscese, perché il tuo animo è implacabile. Ma se temi in cuor tuo qualche divina profezia, se da parte di Zeus tua madre ti ha rivelato qualcosa, manda me allora, e presto, con tutti i Mirmidoni, se mai io possa portare luce di salvezza agli Achei. Le tue armi dammi, da portare sulle mie spalle, e i Troiani mi scambieranno per te e si daranno alla fuga, mentre i figli degli Achei, sfiniti, avranno respiro: ne basta poco, in battaglia. Sarà facile allora per noi, freschi di forze, respingere gli uomini stanchi di lotta verso la città, lontano dalle tende, lontano dalle navi' ${ }^{49}$.

\section{Passiamo al $\$ 2$ della Declamazione coriciana:}

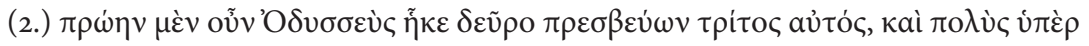

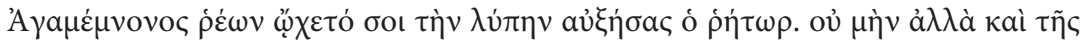

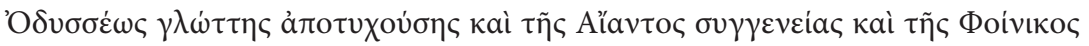

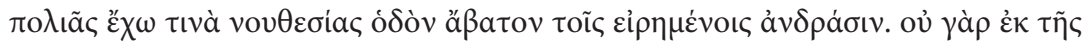

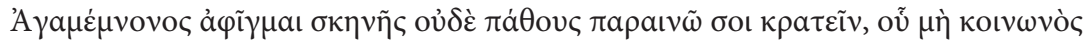

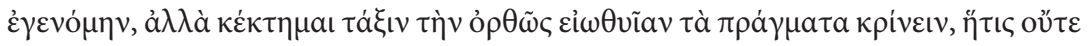

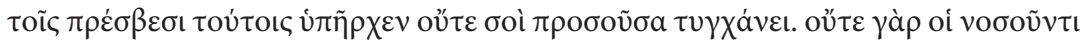

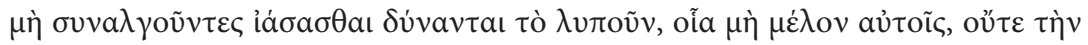

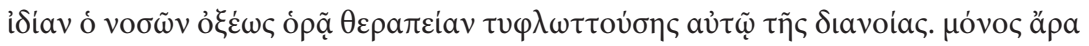

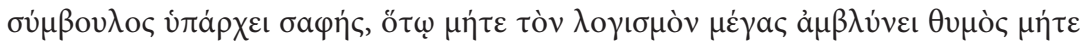

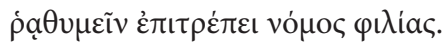

2. «Poco fa, dunque, Odisseo è venuto qui, terzo di un'ambasceria ${ }^{50}$, e discorrendo copiosamente in difesa di Agamennone, quel maestro d'eloquenza si è allontanato

49. Hom., Il. 16, 20-45.

50. L'ambasceria, cui si fa riferimento, è quella di Hom., Il. 9, 162-642, condotta da Odisseo, Fenice e Aiace. Non sicuramente a caso Coricio tiene a precisare, fra i tre, la presenza di Odisseo quale 
dopo aver accresciuto la tua sofferenza. Ciò nonostante, pur non avendo avuto successo la lingua di Odisseo né la parentela di Aiace né la chioma canuta di Fenice, io dispongo di una via di ammonimento inaccessibile agli uomini appena citati. Io, infatti, non sono giunto dalla tenda di Agamennone ${ }^{51}$, né ti esorto a dominare su un sentimento di cui non fui partecipe, ma ho assunto la posizione abituale di giudicare correttamente le azioni, posizione che non era nelle facoltà di questi ambasciatori e che neppure tu ti trovi a rivestire. Né, infatti, coloro che non condividono la sofferenza di chi è malato possono curare il dolore, dal momento che non è una loro preoccupazione, né chi è malato vede chiaramente la propria terapia, perché la sua capacità di comprendere è accecata. Uno solo, dunque, è consigliere preciso, quello cui né una grande rabbia offusca il raziocinio né la legge dell'amicizia consente di essere indolente ${ }^{52}$.

Se facciamo un rapido confronto tra i due, possiamo vedere che il Patroclo iliadico tocca solo alcuni dei punti che, in effetti, Coricio amplierà ${ }^{53}$ :

a) captatio benevolentiae tramite il riconoscimento del ruolo di superiorità che l'amico ricopre e che gli dovrebbe imporre di non adirarsi;

b) breve ma intensa descrizione della disperazione degli Achei con riferimento ai capi feriti;

c) auspicio di non provare mai egli stesso un desiderio di rabbia così devastante;

d) negazione retorica della sua ascendenza mortale e divina, con riferimento agli elementi naturali legati in qualche modo ai genitori e invocati come i loro "sostituti" - Peleo richiama le rocce aspre del monte Pelio, mentre Teti, ninfa marina, le acque azzurre del profondo mare ${ }^{54}$;

e) accenno a un misterioso oracolo di cui l'avrebbe informato la madre ${ }^{55}$;

principale ambasciatore: egli, come diremo fra poco, è il primo eroe a prendere la parola e a dire le cose più irritanti per Achille. Coricio, per il resto, si discosta dall'ordine dei discorsi quali sono riportati nell'Iliade - qui, dopo Odisseo, il primo a prendere la parola era stato, infatti, Fenice, seguito da Aiace -, conformandosi in ciò al suo maestro Procopio di Gaza, che nell'etopea di Fenice (op. VII, 2 Amato) presenta anche lui il medesimo ordine di comparsa. La scelta è probabilmente voluta, in quanto Coricio offrirà in seguito ampio spazio al personaggio di Fenice.

51. Patroclo, infatti, non faceva parte dell'ambasceria presso Achille.

52. Chor., op. XXXVIII (= decl. 10), p. 438, 3-18 F. / R.

53. Tralasciando qui tutta la questione relativa allo schema tipico della supplica iliadica, già più volte analizzato: vd., ad es., Aubriot 1984a.

54. Il riferimento all'ascendenza divina serve ad avvalorare ancora di più il dissenso di Patroclo dalle scelte dell'amico, così come il fatto che l'eroe definisca Achille generato da un oggetto inanimato. Questo spunto fortemente critico, nell'Iliade, da parte di Patroclo, viene da Coricio molto limato, se non quasi ignorato. Non sulle lacrime né sulle espressioni di rabbia, infatti, il retore vuole impostare la sua opera persuasiva.

55. Questo ipotetico vaticinio, che è presente nei Poemi del Ciclo e, in particolare, nei Cypria e nell'Etiopide, è accennato altresì nell'Iliade, in quanto appartiene alla tradizione orale preomerica: 


\section{f) esortazione a cedergli le armi e a mandarlo a condurre i Mirmidoni al posto} suo.

Il discorso iliadico, inoltre, occupa soltanto i vv. 21-45 del canto XVI ed è da immaginarsi recitato tra le lacrime e i lamenti dell'eroe.

La declamazione, invece, si mette da subito in antitesi con il testo omerico, ribadendo - da parte di Patroclo - quelle che sono le differenze rispetto all'ambasceria di Hom., Il. 9.162-642, condotta da Odisseo, Fenice e Aiace. Il retore spiega dunque, commentando il testo omerico, le ragioni più profonde del fallimento della missione dei tre ambasciatori. Non a sproposito né sicuramente a caso Coricio precisa, fra tutti, il nome di Odisseo quale principale rappresentante del messaggio di Agamennone. Mentre, nell'Iliade, Nestore, cavaliere Gerenio, aveva assegnato, difatti, un posto di preminenza al saggio Fenice ${ }^{56}$, dopo uno strano e discusso gioco di sguardi fra Aiace e Fenice, era stato il re di Itaca a prendere la parola per primo nella tenda. Il suo è il primo dei tre discorsi e il più fastidioso per le orecchie di Achille. Coricio fa allora ricordare al suo Patroclo

tale sostrato, ben noto a Omero, pare aver influenzato Il. 11, 794-803. Dall'epica successiva e dalla tradizione scoliastica sappiamo che sono molti gli oracoli che scandiscono la vita del Pelide: quando Achille aveva nove anni, l'indovino Calcante predisse che Troia non avrebbe potuto essere presa senza l'aiuto del giovane. Allora, Teti - o, secondo una variante del mito, Peleo -, venuta a conoscenza della profezia, sottrasse il giovane alle cure di Chirone e lo portò a Sciro, presso il re Licomede. Qui lo fece vestire con abiti femminili e lo fece vivere insieme alle figlie del re, fra le quali sposò Deidamia, con cui generò Neottolemo. Questo oracolo aleggia più volte nell'Iliade, quando Teti si rivolge ad Achille ricordandogli che gli dèi hanno decretato per lui una vita breve, ma gloriosa (Hom., Il. 1, 414-418; 18, 9-11). Nel riassunto di Proclo (Chrest. 185-190 : 186-187 Severyns : kaì @étıৎ

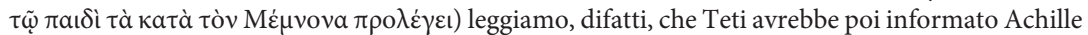
della sorte che attendeva Memnone e che avrebbe dato origine, conseguentemente, alla sua. Sulla fine tragica di Achille circolavano difatti due versioni: una, lo vedeva cadere in un'imboscata dei Troiani il giorno delle nozze con Polissena, mentre l'altra - qui sottintesa - sembrava indicare che Eos e Teti, madri rispettivamente di Memnone e di Achille, dopo aver invano cercato di convincere Zeus a salvare ciascuna il proprio figlio, dovettero assistere alla pesa delle anime dei due eroi. Il destino del giovane Etiope è segnato: dopo aver ucciso Antioco, figlio di Nestore e caro amico del Pelìde - cui in Hom., Il. 18, 17ss annuncia la morte di Patroclo e ne piange insieme con lui -, Memnone viene ucciso. Subito dopo però, nell'assalto che l'eroe compie a Troia, viene ucciso da una freccia scagliata da Paride guidato da Apollo. Cfr. Severyns 1928, 285-291; 318-320. Può essere interessante segnalare che questo oracolo, pur avendo dei veri e propri strascichi nell'Iliade, viene chiamato in causa da Coricio in maniera quasi incoerente solo per introdurre il riferimento ad una leggenda pur non strettamente attinente al motivo dell'allontanamento dell'eroe dal conflitto. Il riferimento al travestimento di Achille a Sciro, smascherato solo grazie al rumore delle armi, sembra ritornare in Coricio al $\$ 75$ (Chor., op. XXXVIII [= decl. 10], p. 475, 20-476, 2 F. / R.) : 'A $v \alpha \lambda \dot{\alpha} \beta \omega \mu \varepsilon v$,

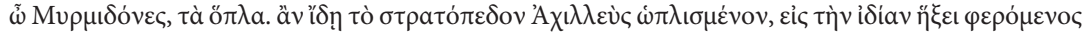

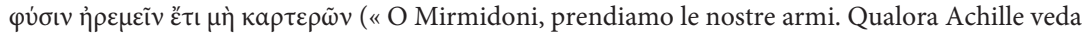
l'esercito con l'armi indosso, tornerà d'un balzo al suo istinto naturale, non resistendo più »).

56. Hom., Il. 9, 167-169: "Vadano quelli che sceglierò io stesso : il capo sia Fenice, amato da Zeus, e poi il grande Aiace e il glorioso Odisseo ». 
solo il discorso del Laerziade, specificando che era riuscito ad aumentare ancora il dispiacere dell'offeso. Il suo personaggio intende allora partire da qui, da quella che subito dopo chiama la «lingua di Odisseo $»^{57}$, passando per la parentela con Aiace Telamonio (cugino di Achille) ${ }^{58}$ e per la chioma di Fenice ${ }^{59}$. Coricio, però, in questo elenco si discosta dall'ordine dei discorsi iliadici: dopo Odisseo, aveva infatti parlato Fenice, seguito, infine, da Aiace. La scelta è molto probabilmente

57. Se Omero è stato già considerato dagli antichi come il padre della retorica (cfr. Plat., Phaedr. 261b 6-8; Ion 54ob; Quint., inst. 2, 17, 6-8), fu in particolare Odisseo ad essere considerato il più efficace fra gli eroi-oratori (cfr. Varrone in Gellio, 6, 14, 7 ; Cic., Brut. 40). Descritta da Omero stesso come dirompente nel celebre passo della Teicoscopia (Hom., Il. 3, 216-224) l'oratoria di Odisseo è il risultato della perfetta padronanza della tecnica retorica: proprio in riferimento a questo passaggio, infatti,

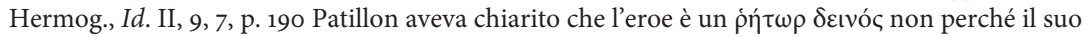
discorso sia terribile, ma, piuttosto, in virtù della sua efficacia. Non per nulla, il Laerziade è uno dei personaggi che parlano di più nel poema (Hom., Il. 2, 190-197; 200-206; 246-264; 284-332; 4 , 350-355; 9, 225-306 ; 11, 404-410). Sul contenuto e le modalità del discorso di Ulisse nell'economia dell'ambasceria, vd. infra, p. 167. Su Omero quale maestro di retorica e sull'oratoria dei suoi eroi si vedano, tra gli altri, Schöpsdau 1969, 56-86, e Dentice di Accadia Ammone 2012, 17-27.

58. Il Grande Aiace, figlio di Telamone e di Peribea, veniva da Salamina. Secondo solamente ad Achille per valore e forza (Hom., Il. 3, 226-271; 7, 279-80), stando ad una tradizione posteriore ad Omero era anche cugino di Achille: Telamone viene spesso presentato, difatti, come fratello di Peleo e amico di Eracle, con cui distrusse una prima volta Troia, governata da Laomedonte. Nell'Iliade, l'ultima delle tre ambasciate ad Achille è proprio quella di Aiace. L'eroe fa sfoggio di una retorica che, sinteticamente e seguendo Dentice di Accadia Ammone 2012, 198-202 (in particolare 200), potremmo chiamare "cameratesca" (Hom., Il. 9, 624-642). L'astuzia di Aiace è quella di non rivolgersi direttamente ad Achille quando pronuncia le parole più virulente. Il brusco passaggio dalla terza alla seconda persona avviene solo nel momento della supplica, quando il Telamonio chiede all'amico di non curarsi di uno solo, ma di tutto il popolo acheo. I due eroi, benché legati dal sangue, sono su due piani antitetici: per lo sbrigativo e pragmatico Aiace, sette donne sono sicuramente più preziose di una sola. Nella sua ottica, non c'è spazio per la possibilità che Achille sia innamorato di Briseide e così egli non può comprendere pienamente la gravità del torto che l'amico ha subito. Tuttavia, è solo dopo l'intervento di Aiace che il Pelìde promette di tornare a combattere qualora Ettore dovesse arrivare a minacciare le sue navi; se, dunque, fino ad allora non sventerà la tremenda carneficina dell'esercito, non esclude più di rientrare in guerra (Hom., Il. 9, 649-655). Su Aiace, vd. Girard 1905; Trapp 1961; O’Higgins 1989; Greco 2002; Greco 2005; Wathelet 2008; Scafoglio 2016.

59. Oltre agli insegnamenti di Chirone in campo musicale e medico, Achille apprese l'arte oratoria da Fenice. Quest'ultimo era arrivato esule a Ftia dopo essere stato accusato dalla concubina di suo padre, Amintore, di aver tentato di violarla. Fenice, per punizione, fu accecato e al tempo stesso colpito dalla maledizione di non poter mai generare figli; fosse vera o falsa l'accusa della donna, Fenice figli non ne ebbe mai. Tuttavia, nella tranquillità della casa di Peleo, egli fu incaricato della custodia del giovane Achille, che gli si affezionò profondamente. Peleo, inoltre, restituitagli la vista grazie all'arte di Chirone, lo elesse re del popolo dei Dolopi. Gli accenni, nel discorso di Fenice ad Achille, al fosco dramma familiare che condizionò da ragazzo la sua vita così come alla fuga da casa e alla nuova vita a Ftia consentono a Fenice di rivolgersi all'allievo e amico come ad un «caro figlio » (Hom., Il. 9, 437, 444), con cui ha da spartire ricordi infantili anche molto dolci (Hom., Il. 9, 485-495). Su Fenice, cfr. Aubriot 1984b e, da ultimo, Guidorizzi 2012. 
anche qui voluta: il retore vuole tenere Fenice per ultimo, in evidenza, perché il suo nome tornerà nel corso della $\mu \varepsilon \lambda \dot{\varepsilon} \tau \eta$.

$\mathrm{Ma}$, cosa più importante di tutte, Coricio fa rivendicare a Patroclo la propria autorità a dirimere la questione: egli snocciola quelli che sono i punti a favore del giovane, i motivi che lo renderebbero il più idoneo a persuadere l'amico. In prima istanza, egli parla senza ingannare l'amico; infatti, proprio di inganno è questione nell'Iliade. Le acerbe risposte di Achille ai tre ambasciatori erano state la reazione allo sdegno per i tentativi che questi avevano messo in atto per circuirlo. Anche quegli ambasciatori, però, erano tre compagni, tre amici, ma erano anche stati inviati da Agamennone. In particolare, rivolgendosi ad Odisseo, Achille aveva detto: «odio, come odio le porte di Ade, colui che altro dice e altro cela nell'animo ${ }^{60}$. Come ha notato Dentice di Accadia, «il fallimento retorico non va cercato allora nel tipo di argomenti scelti, di per sé validi, né nel modo in cui essi sono avanzati, ma piuttosto in ciò che Odisseo non dice. Il suo discorso non contiene alcuna ammissione della colpevolezza di Agamennone, nessuna condanna dell'oltraggio perpetrato ai danni del Pelìde ${ }^{61}$.

Patroclo, difatti, non è stato mandato in seguito all'assemblea nella tenda di Agamennone: egli è profondamente super partes, perché agisce in modo autonomo nel suo rivolgersi all'amico, e non perché è stato assoldato dal capo degli Achei ${ }^{62}$. Come già detto poco fa, la posizione "tendenziosa" degli altri tre ambasciatori era stata una delle ragioni del fallimento della missione.

Coricio, però, nasconde strategicamente il contenuto del dialogo intercorso nel canto XI dell'Iliade, tra il giovane e Nestore, per passare invece subito ad un altro $\operatorname{argomento}^{63}$. La declamazione continua segnalando che Patroclo ha partecipato

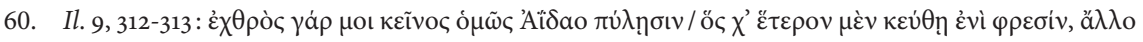

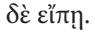

61. Dentice di Accadia Ammone 2012, 183.

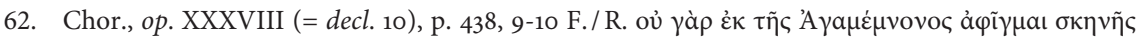
( «Io, infatti, non sono giunto dalla tenda di Agamennone»).

63. Il lungo discorso di Nestore nel canto XI (Hom., Il. 11, 656-803) è uno degli snodi fondamentali del poema: dopo aver abbondantemente illustrato la disastrosa situazione dello schieramento Acheo ed aver ricordato (Il. 11, 780-793) a Patroclo le raccomandazioni del padre Menezio sottolineando come il giovane ne abbia perduto il ricordo, il re di Pilo propone l'unica soluzione possibile. Achille, per quanto irato, dovrebbe concedere almeno a Patroclo di combattere al posto suo, rivestito delle sue armi, così da incutere terrore nei Troiani e infondere coraggio ai Mirmidoni. Ebbene, come si vede, è proprio grazie a questo discorso che Patroclo, nel canto XVI, cercherà di persuadere l'amico non a tornare a combattere, ma a mandare lui : è proprio questo atteggiamento che Coricio, nella declamazione, condanna. Il tema delle promesse dimenticate è, tra l'altro, anch'esso ripreso dal retore: nel $\$ 5$ (Chor., op. XXXVIII [= decl. 10], p. 440, 1-3 F./R) egli fa dire al suo Patroclo:

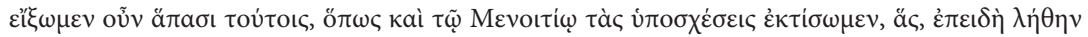

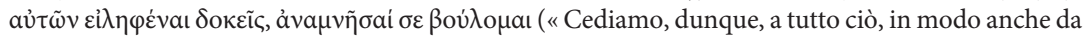
soddisfare le nostre promesse a Menezio, delle quali desidero rinnovarti il ricordo, giacché sembri 
- insieme, ricordiamolo, a tutti i Mirmidoni - dell'ira dell'amico, perché, come lui, si è sentito offeso dall'atteggiamento ingiurioso di Agamennone. Per spiegare e legittimare, appunto, il suo ruolo di consigliere perfetto, Patroclo si presenta, allora, come il solo atto a dirimere la questione ${ }^{64}$. A differenza di Odisseo, nel proseguire il suo discorso, egli affronta il nodo centrale della questione: l'offesa c'è stata ed è avvenuta sotto gli occhi di tutti; per questo motivo Achille ha fatto bene ad andarsene in un primo tempo. Tuttavia, essendo trascorso ormai molto tempo, l'amico deve rassegnarsi a lasciare da parte la sua ostilità ed il suo risentimento.

Meritano un'attenzione particolare i punti che presentano rielaborazioni del testo omerico e che, dunque, tradiscono veri e propri clins d'œil a quella parte del pubblico più accorta.

Per esempio, alla fine del $\$ 4$ :

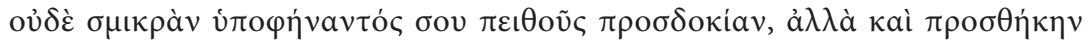

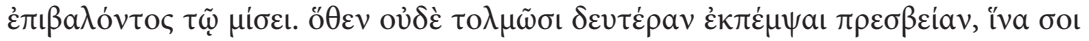

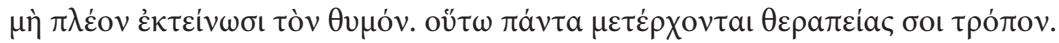
« tu non hai dato la minima impressione di essere persuaso, anzi hai anche accresciuto il tuo odio. Per questo motivo, essi neppure osano inviare una seconda ambasceria col fine di non tendere ulteriormente la tua ira. Così essi si prodigano in ogni modo per servirti ${ }^{65}$.

Qui, in realtà, Coricio commette un errore o volutamente annulla quello che è stato il parziale risultato ottenuto dall'ambasceria nel canto IX. In una sorta di crescendo, infatti, Achille - che con la propria risposta ad Odisseo aveva ancora confermato fermamente l'intenzione di partire il mattino dopo facendo vela verso $\mathrm{Ftia}^{66}$-, sembrava lasciare aperto lo spiraglio di rimandare la decisione della partenza dopo una buona notte di sonno. Così, almeno, promette al piangente Fenice ${ }^{67}$.

averle dimenticate»). Se la "corta memoria" di Patroclo, come abbiamo visto, era stata criticata da Nestore, sempre nell'Iliade, Odisseo, nel corso della sua ambasciata ad Achille (Il. 9, 252-259) gli aveva rammentato gli ammonimenti di Peleo. Per un'analisi di tale procedimento retorico-letterario, vd.

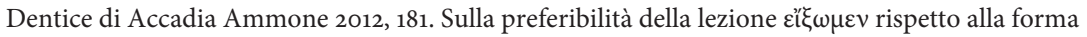
$\dot{v} \pi \varepsilon \dot{\xi} \xi \omega \mu \varepsilon v$ scelta da F. / R., vd. ora Amato 2016c.

64. Questa presentazione di sé, corroborata subito dopo dal paragone con la situazione dei malati, è indispensabile. Infatti, nell'Iliade, ogni oratore è solito presentarsi, adducendo spesso come argomento un episodio relativo alle proprie esperienze passate o al mito (vd. discorsi di Nestore - $\mathrm{Il}$. 11, 656-803 - e Fenice - Il. 9, 434-605).

65. Chor., op. XXXVIII (= decl. 10), p. 439, 15-19 F./R.

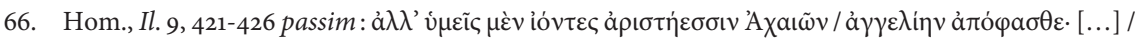

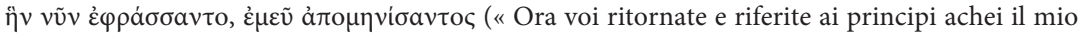
messaggio: [...] io persisto nell'ira").

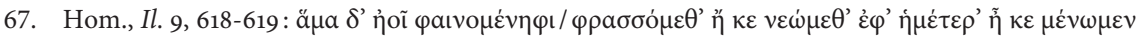
(« domani all'alba decideremo se tornare a casa o rimanere»). 
Ma non basta: dopo l'ultimo intervento, rispondendo ad Aiace, il Pelide si era impegnato a tornare a combattere, qualora Ettore fosse arrivato a minacciare le sue navi; se, dunque, fino ad allora non sarebbe stata sventata la tremenda carneficina dell'esercito, egli non esclude più di rientrare in guerra ${ }^{68}$.

Un altro esempio interessante si trova al $\$ 11$. In questa sezione, Patroclo, ricordando come, nella lite tra Agamennone e Achille, anche quest'ultimo avesse risposto alle ingiurie, macchiandosi a sua volta di colpa, dice:

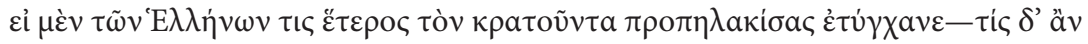

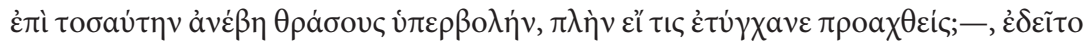

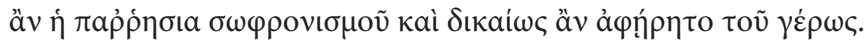

«Se, difatti, un altro fra i Greci avesse per caso coperto di fango chi detiene il potere - ma chi avrebbe potuto azzardare un tale eccesso di audacia, se non qualcuno che vi fosse stato spinto? -, la sua franchezza avrebbe richiesto un castigo e a buon diritto egli sarebbe stato privato del premio ${ }^{69}$.

A ben vedere, però, esempi ce ne sono nell'Iliade. In questo caso, dunque, si prospettano due ipotesi differenti: da un lato Coricio, sapendo che il Patroclo iliadico non ha ascoltato molte assemblee dei capi e in particolare quella del canto IX, non può far dire al suo personaggio cose di cui non è a conoscenza; dall'altro lato - e sembrerebbe l'ipotesi più probabile, oltre che la più accattivante - il Patroclo coriciano, vero retore esperto, seleziona accuratamente il materiale a sua disposizione e sottopone ad Achille, sì da persuaderlo, soltanto quegli argomenti che gli sembrano più pertinenti. Difatti, mi pare che l'esempio più calzante di una situazione come quella qui prospettata vada proprio individuata nell'assemblea collettiva del canto IX ${ }^{70}$, nella quale Diomede - che, benché offeso da Agamennone nel canto IV ${ }^{71}$, non aveva osato rispondere e mancargli così di rispetto - decide di opporsi violentemente al capo supremo, dopo la proposta di abbandonare la guerra e partire ${ }^{72}$.

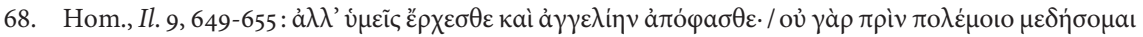

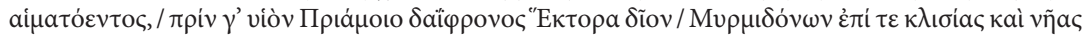

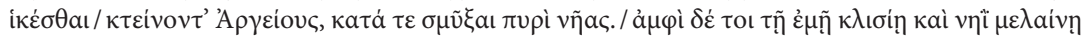
( « Ma voi andate e riferite il messaggio : io non mi curerò della battaglia cruenta prima che il figlio del valoroso Priamo, prima che Ettore glorioso facendo strage di Achei sia giunto alle tende e alle navi dei Mirmidoni e abbia dato fuoco alle navi; vicino alla mia tenda e alla mia nave nera, io fermerò Ettore e la sua furia di guerra»).

69. Chor., op. XXXVIII (=decl. 10), p. 443, 9-13 F./ R.

70. A tale assemblea farà seguito una seconda, riservata ai soli capi, che sfocerà nella decisione di inviare l'ambasceria.

71. Hom., Il. 4, 370-421.

72. Hom., Il. 9, 32-49. 
Un caso similare è quello che si trova poco più avanti $(\$ 14)$, quando Patroclo, nel descrivere la scena pietosa del comandante chino e piangente in mezzo ai soldati, ricorda come Nestore si sia frapposto per fermare la fuga precipitosa degli Achei.

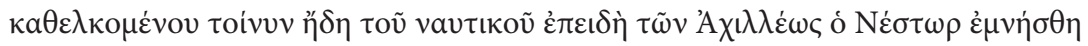

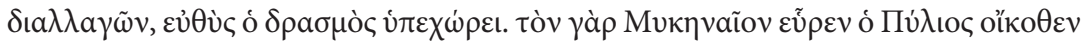

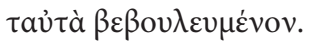

«Pertanto, pur già tirate le navi in acqua allorché Nestore fece menzione delle riconciliazioni con Achille, la fuga cominciò immediatamente a scemare. Difatti, il Pilio scoprì che il Miceneo aveva deciso assolutamente di perseguire la medesima linea di condotta $»^{73}$.

Patroclo, nuovamente, modella la materia a suo piacimento così da essere più convincente. Ben sapendo che l'obiezione mossa da Achille riguarderebbe la genuinità d'animo del comandante supremo nel proporre finalmente una riconciliazione, egli cerca in tutti i modi di presentare quello che era stato - evidentemente - un suggerimento di Nestore come un'autonoma iniziativa dell'Atride ${ }^{74}$. Come se non bastasse, l'eroe, nel paragrafo successivo, continua a difendere l'operato di Agamennone, modificando la realtà dei fatti. Risulta altresì assai intrigante l'eliminazione di quelli che nell'Iliade costituivano due momenti distinti, ovvero le due diverse assemblee. Nestore, infatti, dopo la replica a Diomede, aveva proposto che tutti prendessero congedo e andassero a mangiare e a riposarsi ${ }^{75}$, ma aveva poi fatto convocare un'assemblea privata, il "Concilio ristretto", proponendo soltanto in questa cornice la celebre ambasceria.

L'abilità elocutoria del retore si manifesta, quindi, nel ribattere ad altri due punti fermi dell'ira iliadica: la questione relativa ai doni, proposti da Agamennone per il tramite di Odisseo, che avevano - in parte - aumentato l'offesa, e la sorte toccata a Briseide, una volta portata via a forza dalla tenda di Achille.

$\mathrm{Al} \S 16$, per esempio, Patroclo conferma l'opinione corrente di un Agamennone avido oltremisura, ma per ribaltarla e usarla così a vantaggio della sua argomentazione:

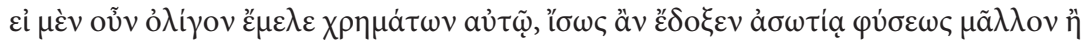

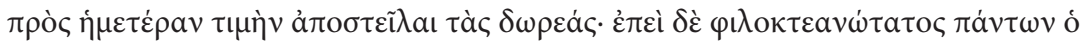

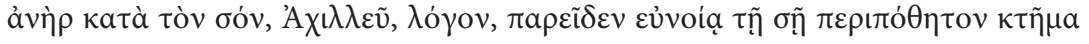

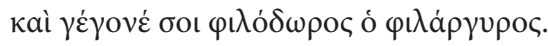

« Ebbene, se si fosse curato poco delle ricchezze, forse avrebbe deciso di elargire i doni per la prodigalità della propria natura piuttosto che per rispetto verso di noi;

73. Chor., op. XXXVIII (= decl. 10), p. 445, 4-7 F./R.

74. Cfr. Il. 9, 92-161.

75. Cfr. Il. 9, 65-78. 
ma poiché egli è, stando alle tue parole, o Achille, l'uomo 'più avido di guadagno di tutti' ${ }^{76}$, egli ha visto nella tua benevolenza un bene grandemente bramato, sicché è divenuto per te generoso l'avaro $»^{77}$.

Patroclo cambia le carte in gioco ancora una volta: al fine di persuadere il suo amico, sfrutta magistralmente la questione dei doni. Nell' Iliade, l'accenno ai $\delta \tilde{\omega} \rho \alpha$ da parte di Odisseo ${ }^{78}$ altro non faceva se non suscitare la rabbia di Achille, che li aveva trovati insultanti. Invece, con l'astuzia retorica, il figlio di Menezio gli impedisce di formulare l'accusa di avarizia o quella di non aver cercato di ripagare il suo onore ${ }^{79}$.

In ambito retorico, però, il problema del giuramento di Agamennone di non aver mai sfiorato la fanciulla dopo averla fatta prelevare dalla tenda del rivale, aveva avuto una discreta fortuna. Fra tutti, ricordiamo la breve Etopea di Briseide (=Etopea 1 Amato) di Severo di Alessandria ${ }^{80}$, che rappresenta le immaginarie parole che la fanciulla avrebbe rivolto a se stessa dopo essere stata trasferita lontano da Achille, e il Discorso 56 di Dione di Prusa, in cui è questione dei limiti del potere di Agamennone ${ }^{81}$.

76. Cfr. Hom., Il. 1, 122.

77. Chor., op. XXXVIII (= decl. 10), p. 446, 6-11 F./R.

78. Può essere utile ricordare che il lungo catalogo dei beni sciorinato da Odisseo ripeteva le parole di Agamennone, con la mutazione di alcune forme verbali. Cfr. Hom., Il. 9, 122-157 = Il. 9, 264-299.

79. Bisogna però sottolineare un altro aspetto : nei poemi omerici è fondamentale un codice di valori eroici, le cui esigenze, naturalmente, non erano più comprese né tantomeno accettate nell'epoca di Coricio. Il motivo dell'ira nel poema omerico è - di fatto - uno solo : il grave danno inferto al suo onore è scaturito dalla sottrazione di un bene assegnatogli dalla comunità guerriera tutta ed esso non può essere cancellato neppure con una miriade di doni stupefacenti. Se questo fosse possibile, Achille confermerebbe un ruolo di fatto subordinato a quello del comandante supremo: questi, di conseguenza, come lo ha ferito una volta, sapendo che potrebbe sempre "ricomprarlo" qualora avesse necessità del suo braccio con fastosi donativi, sarebbe tranquillamente libero di oltraggiarlo un'altra volta. La questione di Briseide, in questo quadro, è una conseguenza, ma secondaria.

80. Per la analisi del testo, delle sue fonti e del suo stile, cfr. Amato \& Ventrella 2009, 63-70.

81. Per l'Agamennone (or. 56) di Dione di Prusa, cfr. Deroma 2013-2014. In particolare, nel $\$ 14$ dello stesso discorso Agamennone, dopo una lunga citazione da Il. 9, 106-112, Dione, nel tentativo di

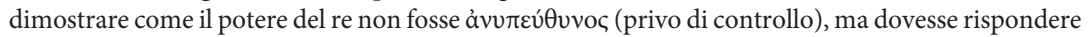
ad altre istanze (i. e. consiglio ristretto dei capi e consigli privati del saggio Nestore), parafrasa proprio il discorso dell'Atrìde, che propone il risarcimento ad Achille: кaì vì $\Delta$ ía $\gamma \varepsilon$ oủ Hóvov

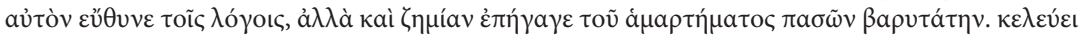

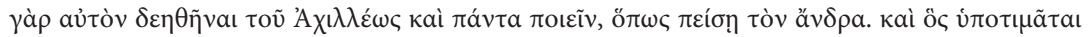

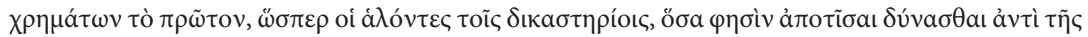

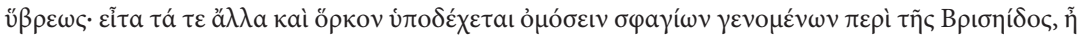

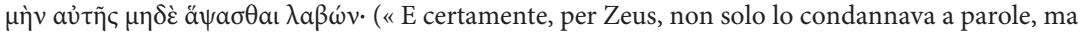
gli inflisse anche la punizione più dura di tutte per il suo errore. Ordina infatti a costui di pregare Achille e di fare ogni cosa per persuaderlo. E dapprima l'Atrìde, come coloro che sono dichiarati colpevoli dai tribunali, propone per se stesso una pena minore in ricchezze, quante dice di essere in grado di ripagare in cambio della sua arroganza; in seguito promette, tra le altre cose, anche 
La questione del giuramento del re è uno dei temi più scottanti anche della declamazione coriciana e il retore di Gaza la affronta in modo intelligente, quasi rispondendo alle violente parole che nel canto IX Achille aveva pronunciato in merito al suo totale disinteresse per la fanciulla. Nella sua invettiva ad Odisseo, ma diretta in realtà ad Agamennone, il Pelìde era arrivato difatti ad affermare: «ai re e agli eroi concedeva i doni d'onore, ed essi li conservano intatti; a me solo fra gli Achei l'ha tolto, la sposa diletta che ora ha con sé. Dorma con lei e goda! [...] $»^{82}$.

Coricio, per bocca del suo Patroclo, guida l'ascoltatore attraverso una serie di prove che dimostrano che non ci deve essere un motivo per dubitare della buona fede di Agamennone, il quale, avendo già più volte sperimentato la vendetta divina, non avrebbe mai il coraggio di commettere spergiuro.

Senza soffermarci su tutti gli altri casi in cui il retore gazeo rielabora la materia iliadica, possiamo ora accingerci a tracciare alcune conclusioni.

\section{Conclusioni}

Alla luce del discorso qui delineato, nel Patroclus coriciano Omero e la sua opera non costituiscono, dunque, autorità indiscutibili, ma offrono lo spunto per la creazione di una versione alternativa, di un vero e proprio proficuo scambio, anche sotto forma di sfida con il modello ${ }^{83}$. Un po' alla maniera di Dione, che ribalta nel suo discorso Troiano le sorti della guerra di Troia, Coricio modifica uno degli snodi

che dopo aver fatto un sacrificio avrebbe fatto un giuramento riguardo Briseide, di non averla in realtà mai toccata, pur avendola sottratta»). Mi sembra altresì opportuno segnalare come pertinente anche il paragone che il Prusense instaura fra Agamennone e l'imputato a un processo. Per quanto riguarda il verso citato da Coricio, cfr. Il. 9, 133, 275; 19, 258-265.

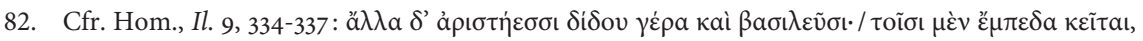

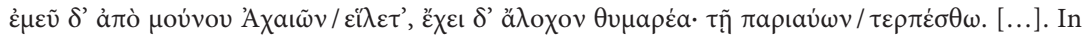
questo passaggio iliadico, Achille considera Briseide non un semplice bottino di guerra, un $\gamma \dot{\varepsilon} \rho a \varsigma$,

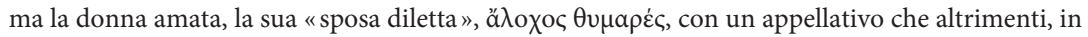
Omero, è usato soltanto per Penelope (cfr. Od. 23, 232). Una definizione così forte del valore di Briseide per Achille non può essere però ricondotta solamente alla necessità del successivo paragone di Elena per Menelao. In tale ruolo, risiede la cifra del rapporto privilegiato che lega il Pelide alla figlia di Brise, che non è in alcun modo paragonabile alle altre donne catturate o ricevute in dono. Sarà Briseide a piangere sul corpo di Patroclo, ricordando la promessa che questo le aveva fatto di accompagnarla a sposare Achille (Il. 19, 287-300); sarà accanto a lei, che Achille - per l'ultima volta nel poema - si ritirerà a dormire (Il. 24, 675-676). La secca replica di Aiace («ma a te un'ira funesta, implacabile hanno messo in cuore gli dei per una fanciulla, una sola; e ora noi te ne offriamo sette, bellissime e oltre ad esse molti altri tesori» [Il. 9, 636-639]) dimostra che i due eroi, benché legati dal sangue sono su due piani antitetici: per lo sbrigativo e pragmatico Aiace, sette donne sono sicuramente più preziose di una sola. Nella sua ottica, non c'è spazio per la possibilità che Achille sia innamorato di Briseide e così non può comprende pienamente la gravità del torto subito da parte di Agamennone.

83. Cfr., per il caso di Libanio, Webb 2010, 137-138; 152. 
fondamentali del poema: il suo Patroclo e Achille, alla fine della declamazione, potrebbero prendere insieme le armi, convinti che - come nell'augurio di Achille nel canto XVI - « noi due scampati alla strage, noi due soli potessimo sciogliere i sacri veli di Troia! $»^{84}$. Il $\mu$ ṽ 0 o viene modificato, alterato, ma rispettato nella sua verosimiglianza: Coricio e il suo pubblico conoscono la "storia", ma tutti seguono il discorso di Patroclo in questo processo di convincimento, che passo dopo passo porterà Achille a imbracciare le armi ${ }^{85}$.

Proprio il pubblico, allora, sarà da ricercare in un gruppo di allievi che, magari, nel corso dell'anno, si sono esercitati nella lettura e nel commento di alcuni canti di Omero sotto la guida del retore ${ }^{86}$. Ora, giunti a fine percorso, il retore vuole mostrar loro - perché no, in un'occasione di festa - la vera forza della retorica applicata. La materia iliadica diventa così - probabilmente dopo un commento specifico al testo contenuto ideale per una performance di tipo differente, atta a veicolare un messaggio positivo e in linea con l'etica professata all'interno del contesto scolastico gazeo. Oltre a veicolare, come già detto, importanti elementi culturali identitari marcatamente ellenici, Coricio mostra pregi e difetti del più grande e amato eroe dell'epica ${ }^{87}$ e del suo amico : in fin dei conti, sono uomini, dominati da sentimenti anche riprovevoli, ma sui quali, alla fine, prevale sicuramente $\varphi \iota \lambda \alpha v \theta \rho \omega \pi i a$ e $\dot{\epsilon} \pi \varepsilon \dot{\varepsilon} \kappa \varepsilon \iota \alpha$.

Le aspettative del pubblico coriciano sono allora completamente raggiunte, per il tramite di un discorso giudicato - da alcuni - forse un po' troppo lungo: grazie alla seconda delle due dialexeis legate al testo (Chor., op. XXXVII F. / R. [= dial. 23]), difatti, veniamo informati che la recitazione del Patroclus aveva probabilmente richiesto una pausa a metà ${ }^{88}$. Il fatto che il discorso d'intrattenimento sia «indirizzato contro chi ha criticato il fatto che la lunghezza del discorso non è adeguata alla statura del parlante ${ }^{89}$, induce a pensare che Coricio avesse risposto

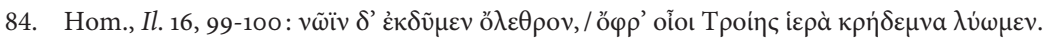

85. Cfr., a questo proposito, l'analisi del Troiano dioneo fatta da Bolonyai 2001 e n. 20.

86. Normalmente, spettava al grammatico introdurre e spiegare Omero, ma era poi con il retore che si potevano riprendere alcuni episodi o tematiche per analizzarle da una angolatura differente. I giovani - che avevano studiato con il $\gamma \rho \alpha \mu \mu \alpha \tau$ retorica soltanto dopo aver acquisito la perfetta capacità di padroneggiare gli esercizi appropriati,

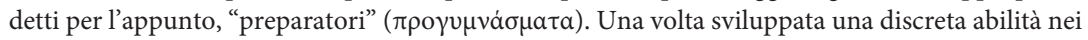
differenti tipi di esercizi, gli allievi dovevano migliorare l'arte della parola in tutte le sue componenti. Cfr. Theon, Prog. 60, 1-10, p. 2, e 72, 22-25, p. 18 Patillon. Vd. anche Robert 2015.

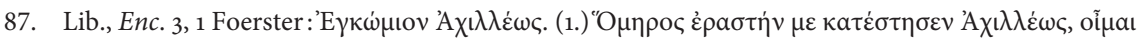

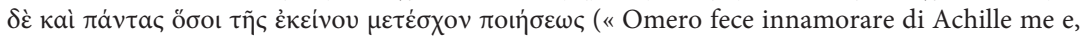
credo, tutti gli altri, quanti hanno avuto a che fare con la sua poesia.»).

88. Questo comporta, di solito, la presenza di una seconda $\delta\llcorner\dot{a} \lambda \varepsilon \xi \xi \varsigma$ legata ad una declamazione. Cfr. Lupi 2014a, 7-10.

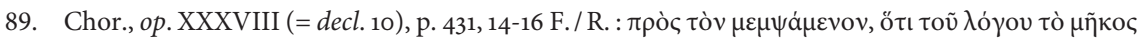

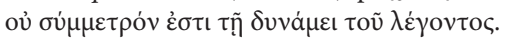


alla critica di chi si lamentava per l'eccessivo spazio concesso alla maschera di Patroclo, personaggio - per l'appunto - praticamente muto nell'Iliade. Niente di più facile, dunque - presupponendo l'attendibilità delle dialexeis, delle hypotheseis e delle note marginali -, che immaginare questa critica rivolta proprio a qualcuno che si era realmente indignato per una così scarsa fedeltà al testo omerico.

Tuttavia, grazie alla sua declamazione, Coricio si inserisce nella lunga scia di retori e maestri che rinnovano le tematiche e i valori del passato epico e glorioso della Grecia - in questo caso, fra gli altri, il sacro valore dell'amicizia - ad uso della collettività. Nel Patroclus non c'è posto per oracoli e predizioni divine, ma gli dei - quando vi si fa ricorso - sono oggetto di esempi positivi, di clemenza e dell'efficacia della preghiera. Il messaggio morale che si impegnano a trasmettere è la lezione che Coricio vuole impartire al suo pubblico - di allievi o meno : l'ira non può mai protrarsi oltre un certo limite.

Si tratti, infatti, di un pubblico meramente studentesco o di uno più eterogeneo con la sua fitta trama - mai banale o pedante - il Patroclus unisce sapientemente critica letteraria e paideia. Una situazione di questo genere testimonia un contatto quotidiano con i grandi autori del passato, richiamati tramite la memoria con dotte citazioni o con allusioni, al fine di sorprendere e affascinare il pubblico, ma anche di instaurare un confronto con gli auctores della storia letteraria.

Matteo Deroma

Université de Nantes 


\section{Riferimenti bibliografici}

AA.VV. (2007), Gaza à la croisée des civilisations, I. Contexte historique et archéologique, Neuchâtel - Genève, Chaman Édition - Musée d'art et d'histoire.

Аmato E. (2009), "The Fortune and Reception of Choricius and of his Works », in Penella 2009, p. 261-302.

Амato E. (ed.) (2010), Rose di Gaza. Gli scritti retorico-sofistici e le Epistole di Procopio di Gaza, Alessandria, Edizioni dell'Orso (Hellenica; 35).

Амato E. (2010a), «Procopio e il dies rosarum : eros platonico, agape cristiana e rappresentazioni pantomimiche nella Gaza tardoantica», in Amato 2010, p. 56-70.

Amato E. (2010b), «Paganesimo e cristianesimo in Procopio di Gaza: su un'incompresa allegoria del vino eucaristico", Athenaeum, 98, p. 503-513.

Amato E. (2012), «Discours figuré et allégorie chrétienne dans l'œuvre “profane” de Procope de Gaza: vin eucharistique et doctrina arcani», in Rolet 2012, p. 209-225.

Amato E. (2014), Procope de Gaza. Discours et fragments, E. Amato (éd., introd. et comm., avec la collaboration d'A. Corcella et de G. Ventrella), P. Maréchaux (trad.), Paris, Les Belles Lettres (CUF; 503).

Амato E. (2015), «Spigolature coricane (I)», Appunti Romani di Filologia, 17, p. 131-144.

Амато E. (2016a), «Spigolature coriciane (II)», Medioevo greco, 16, p. 1-14.

Амато E. (2016b), «Spigolature coriciane (III)», in Montes Cala et al. 2016, p. 277-289.

Амато E. (2016c), «Spigolature coriciane (IV)», Kentron, 32, p. 185-196.

Amato E., Ventrella G. (2009), I progimnasmi di Severo di Alessandria (Severo di Antiochia?), introduzione, traduzione e commento, Berlin - New York, De Gruyter (Sammlung wissenschaftlicher Commentare).

Amato E., Sauterel N. (2010), «L'utilisation de l'histoire dans les déclamations de Lesbonax le Sophiste», in Malosse et al. 2010, p. 47-54.

Amato E., Thévenet L., Ventrella G. (edd.) (2014), Discorso pubblico e declamazione scolastica a Gaza nella tarda antichità: Coricio di Gaza e la sua opera (Atti della giornata di studio, Nantes, 6 giugno 2014), Bari, Edizioni di Pagina (Due punti ; 38).

Amato E., Corcella A., Lauritzen D. (edd.) (2016), L’École de Gaza: espace littéraire et identité culturelle dans l'Antiquité tardive (Actes du colloque international, Paris, Collège de France, 23-25 mai 2013), Leuven, Peeters (Orientalia Lovaniensia analecta, Bibliothèque de Byzantion; 12) (in c.d.s.).

Andorlini I., Bastianini G., Manfredi M., Menci G. (edd.) (2001), Atti del XXII Congresso Internazionale di Papirologia, Firenze, 23-29 agosto 1998, Firenze, Istituto Papirologico «G. Vitelli». 
Aubriot D. (1984a), «Les Litai d'Homère et la Dikè d'Hésiode», REG, 97, p. 1-25.

Aubriot D. (1984b), «Remarques sur le personnage de Phénix au chant IX de l'Iliade», $B A G B$, Lettres d'humanité, 43, p. 339-362.

Bagnall R.S. (ed.) (2009), The Oxford Handbook of Papyrology, Oxford, Oxford University Press.

Bitton-Ashkeloni B., Kofsky A. (edd.) (2004), Christian Gaza in Late Antiquity, Leiden - Boston, Brill (Jerusalem Studies in Religion and Culture; 3 ).

Bolonyai G. (2001), "The Uses of progymnasmata: the case of “refutations" and Dio's Trojan Oration", AAntHung, 41, p. 25-34.

Bоoтн A.D. (1979), «Elementary and Secondary Education in the Roman Empire», Florilegium, 1, p. 1-14.

Browning R. (1975), «Homer in Byzantium», Viator, 6, p. 15-33.

Browning R. (1992), "The Byzantines and Homer», in Lamberton \& Keaney 1992, p. 134-148.

Browning R. (2001), «Education in the Roman Empire», in Cameron et al. 2001, p. $855-883$.

Calboli Montefusco L. (ed.) (2002), Papers on Rhetoric IV, Roma, Herder.

Cameron A., Ward-Perkins B., Whitby M. (edd.) (2001), Cambridge Ancient History, vol. XIV, Late Antiquity: Empire and Successors, A.D. 425-60o, Cambridge, Cambridge University Press.

Cavallo G. (2001), «L'altra lettura. Tra nuovi libri e nuovi testi», AntTard, 9, p. 131-138.

Celentano M.S., Chiron P., Mack P. (edd.) (2015), Rhetorical Arguments. Essays in Honour of Lucia Calboli Montefusco, Hildesheim, Georg Olms Verlag (Europaea Memoria, Reihe I: Studien; 111).

Ciani M.G., Avezzù E. (1998), Omero. Iliade, M.G. Ciani (trad.), E. Avezzù (comm.), Torino, UTET.

Cicu E., Gavini A., Sechi M. (edd.) (2014), Alta Formazione e Ricerca in Sardegna (Atti del convegno di studi Giovani Ricercatori, Sassari, 16 dicembre 2011), Raleigh, Aonia edizioni.

Cipriani G., Tedeschi A. (edd.) (2013), Le chiavi del mito e della storia, Bari, Levante Editori (Kleos. Estemporaneo di studi e testi sulla fortuna dell'antico; 24).

Civiletti M. (2002a), «Mélete: analisi semantica e definizione di un genere», in Calboli Montefusco 2002, p. 61-87.

Civiletti M. (2002b), Filostrato, Vite dei Sofisti, Milano, Bompiani (Il pensiero occidentale).

Corcella A. (2005), "Choriciana », Paideia, 6o, p. 79-93. 
Corcella (2013), "Usi del mito a Gaza», in Cipriani \& Tedeschi 2013, p. 77-99.

Cribiore R. (1993), «A Homeric Exercise from the Byzantine Schoolroom», CE, 68, p. $145-154$.

Cribiore R. (1994), "A Homeric Writing Exercise and Reading Homer in School», Tyche, 9, p. 1-8.

Cribiore R. (1996), «Gli esercizi scolastici dell’Egitto grecoromano: Cultura letteraria e cultura popolare nella scuola», in Pecere \& Stramaglia 1996, p. 505-525.

Cribiore R. (2001a), "School Papyri and the Textual Tradition of Homer », in Andorlini et al. 2001, p. 279-286.

Cribiore R. (2001b), Gymnastics of the Mind: Greek Education in Hellenistic and Roman Egypt, Princeton, Princeton University Press.

Cribiore R. (2009), «Education in the Papyri», in Bagnall 2009, p. 320-337.

Dentice di Accadia Ammone S. (2012), Omero e i suoi oratori. Tecniche di persuasione nell'Iliade, Berlin - Boston, De Gruyter (Beiträge zur Altertumskunde; 302).

Deroma M. (2013-2014), «Il filosofo e il potere: l'A gamennone di Dione di Prusa », RET, 3, p. 103-140.

Deroma M. (2014), «Per un'introduzione al Patroclus di Coricio di Gaza (op. XXXVIII [decl. 10] F. / R.) », in Amato et al. 2014, p. 138-169.

Downey G. (1963), Gaza in the Early Sixth Century, Norman, University of Oklahoma Press (The Centers of Civilization Series; 8).

Dubel S., Favreau-Linder A.-M., Oudot E. (edd.) (2015), À l'école d'Homère. La culture des orateurs et des sophistes, Paris, Éditions Rue d'Ulm, Presses de l'École normale supérieure (Études de littérature ancienne; 24).

DunEAu J.-F. (1971), Les écoles dans les provinces de l'Empire byzantin jusqu'à la conquête arabe (thèse de doctorat), Paris, Université de Paris I.

Foerster R., Richtsteig E. (1929), Choricii Gazaei Opera, recensuit R. Foerster, confecit E. Richtsteig, Lipsiae, in aedibus B.G. Teubneri (BT) (rist. Stuttgart 1972).

GirARD P. (1905), «Ajax fils de Télamon : étude de mythologie héroïque », REG, 18, p. 1-75.

GluCker C.A.M. (1987), The City of Gaza in the Roman and Byzantine Periods, Oxford, British Archaeological Reports (BAR International Series).

Greco A. (2002), «Aiace Telamonio e Teucro. Le tecniche di combattimento nella Grecia micenea dell'età delle tombe a fossa», in Montanari 2002, p. 561-578.

Greco A. (2005), «Omero tra mondo miceneo e arcaico : Aiace Telamonio e gli arcieri locresi », Sileno 31, p. 275-286.

Guidorizzi G. (2012), «Un padre, un figlio e una donna contesa : il caso di Fenice», Index, 40, p. 68-79. 
Hadjittofi F. (2016), «Homer is a Dancer ("O in Amato et al. 2016, p. 151-162.

Kaster R.A. (1983), "Notes on "Primary" and "Secondary" Schools in Late Antiquity", TAPhA, 113, p. 323-346.

Lamberton R., Keaney J.J. (edd.) (1992), Homer's Ancient Readers. The Hermeneutics of Greek Epic's Earliest Exegetes, Princeton, Princeton University Press.

Litsas F.K. (1980), Choricius of Gaza. An Approach to his Work. Introduction, Translation, Commentary (Ph.D. diss.), University of Chicago.

Lupi S. (2009), «Uso del passato nella declamazione 4 di Coricio di Gaza (XVII = decl. 4 F.-R.)», Klio, 91, p. 196-207.

Lupi S. (2010), «Il Milziade di Coricio di Gaza (Or. 17) : rappresentazione retorica di una vicenda storica», Eikasmos, 21, p. 309-326.

Lupi S. (2014a), "Occasione e performance nelle declamazioni di Coricio di Gaza », in Amato et al. 2014, p. 2-19.

Lupi S. (2014b), «Essere uomini, diventare donne: i Lidi di Coricio di Gaza tra storia e riflessione sui meccanismi di costruzione dell'identità individuale», in Cicu et al. 2014, p. 135-149.

Lupi S. (2016), «O p. 267-286.

Malosse P.L., Noël M.-P., Schouler B. (edd.) (2010), Clio sous le regard d'Hermès. L'utilisation de l'histoire dans la rhétorique ancienne de l'époque hellénistique à l'Antiquité tardive (Actes du colloque international de Montpellier, 18-20 octobre 2007), Alessandria, Edizioni dell'Orso (Cardo; 8).

Marrou H.-I. (1994), Storia dell'educazione nell'antichità, Roma, Herder.

Milazzo A.M. (2015), "Riuso mitologico e strategia retorica nel Priamo di Coricio », in Celentano et al. 2015, p. 297-307.

Montanari F. (ed.) (2002), Omero tremila anni dopo, Roma, Edizioni di storia e letteratura (Raccolta di studi e testi; 210).

Montes Cala J.G. (†), Gallé Cejudo R.J., Sánchez Ortiz de Landaluce M., Silva SÁNCHEZ T. (edd.) (2016), Fronteras entre el verso y la prosa en la literatura helenística y helenístico-romana. Homenaje al Prof. José Guillermo Montes Cala, Bari, Levante.

O'Higgins D. (1989), «The Second Best of the Achaeans», Hermathena, 147, p. 43-56.

Patillon M. (1990), Éléments de rhétorique classique, Paris, Armand Colin (Fac. Linguistique).

Patillon M. (1997a), Hermogène, L'art rhétorique (introd., trad. et notes, avec une préface de P. Laurens), Lausanne, L'Âge d'Homme. 
Patillon M. (1997b), Aelius Theon, Progymnasmata, M. Patillon (ed., trad.), Paris, Les Belles Lettres (CUF; 376).

Pecere O., Stramaglia A. (edd.) (1996), La letteratura di consumo nel mondo grecolatino (Atti del convegno internazionale Cassino, 14-17 settembre 1994), Cassino, Università.

Penella R.J. (ed.) (2009), Rhetorical Exercises from Late Antiquity. A Translation of Choricius of Gaza's Preliminary Talks and Declamations, Cambridge - New York, Cambridge University Press.

Pernot L. (200o), La rhétorique dans l'Antiquité, Paris, Le Livre de Poche (Références).

Petit P. (1957), Les étudiants de Libanius. Un professeur de faculté et ses élèves au BasEmpire, Paris, Nouvelles Éditions Latines (Études prosopographiques, I).

RENAUt D. (2005), «Les déclamations d'ekphraseis: une réalité vivante à Gaza au $\mathrm{VI}^{\mathrm{e}}$ siècle», in Saliou 2005, p. 197-220.

Robert F. (2015), «La présence d'Homère dans les Progymnasmata d'époque impériale», in Dubel et al. 2015, p. 73-86.

Rolet A. (ed.) (2012), Allégorie et symbole: voies de dissidence? De l'Antiquité à la Renaissance, Rennes, Presses universitaires de Rennes (Interférences).

Russell D.A. (1983), Greek Declamation, Cambridge, Cambridge University Press.

Saliou C. (2005) (ed.), Gaza dans l'Antiquité tardive. Archéologie, rhétorique et histoire (Actes du colloque international de Poitiers, 6-7 mai 2004), Salerno, Helios (Cardo. Études et textes pour l'identité culturelle de l'Antiquité tardive; 2).

Scafoglio G. (2016), Ajax. Un héros qui vient de loin, Rennes, Presses universitaires de Rennes (in c.d.s.).

SchöPSDAu K. (1969), Antike Vorstellungen von der Geschichte der griechischen Rhetorik, Saarbrücken (Diss. phil. [masch.]).

SChouler B. (2005), "Chorikios déclamateur», in Saliou 2005, p. 117-133.

Schouler B. (2015), «Pour les sophistes, Achille ne fut-il que colère?», in Dubel et al. 2015, p. 87-102.

Severyns A. (1928), Le cycle épique dans l'école d'Aristarque, Liège - Paris, VaillantCarmanne - Édouard Champion (Bibliothèque de la Faculté de Philosophie et Lettres de l'Université de Liège; fasc. XL).

Sivan H. (2008), Palestine in Late Antiquity, Oxford - New York, Oxford University Press.

Stenger J.R. (2016), « Choricius' Unease about Myths or How to Stand Up for the Classics in Gaza», in Amato et al. 2016, p. 1-18.

Telesca C. (2011-2012), «Sull'ordine e la composizione del corpus di Coricio di Gaza», RET, 1, p. 85-109. 
Telesca C. (2013), «Celebrazioni nuziali e performance oratoria negli epitalami di Coricio di Gaza», Medioevo greco, 13, p. 251-271.

Telesca C. (2016), «Erudizione e realtà sociale negli Epitalami di Coricio di Gaza», in Amato et al. 2016, p. 53-72.

Trapp R.L. (1961), «Ajax in the Iliad», CJ, 56, p. 271-275.

Ventrella G. (2011), «Afrodite "gloria del Libano" ? Nota esegetica a Giorgio Grammatico, anacr. 1, 61-64", Eruditio Antiqua, 3, p. 153-16o.

Ventrella G. (2011-2012), «Poesia pagana e simboli cristiani nella Gaza tardo-antica: la "conversione" del mito di Afrodite e della rosa in Giorgio Grammatico ", RET, 1, p. 71-84.

Ventrella G. (2012), «Mito pagano e interpretatio Christiana a Gaza nel VI sec.: l'immagine della rosa fiorita sull'ulivo selvatico in Giorgio Grammatico », Athenaeum, 100, p. 385-404.

Wathelet P. (2008), Les deux Ajax ou Ajax seul dans la tradition homérique et après, texte d'une communication présentée à l'université Charles-de-Gaulle - Lille III le 30 septembre 2008 (PDF).

Webв R. (2010), «Between Poetry and Rhetoric: Libanios' Use of Homeric Subjects in his Progymnasmata», QUCC, 95/2, p. 131-152. 


\section{APPENDICE \\ I DISCORSI ILIADICI NEL PATROCLUS}

\begin{tabular}{|c|c|c|c|}
\hline Canto & Pers. e vv. & Discorso & $\$$ Patroclus \\
\hline \multirow{3}{*}{ IX } & $\begin{array}{c}\text { Od. } \\
225-306\end{array}$ & $\begin{array}{l}\text { Captatio benevolentiae } \\
\text { Disastrose conseguenze dell'ulteriore rifiuto } \\
\text { = muovere alla pietà } \\
\underline{\text { ispirare rabbia verso i nemici }} \\
\text { sviluppare il senso di colpa per i lutti achei } \\
\text { Monito di Peleo dimenticato. } \\
\text { Può rimediare cedendo. } \\
\text { Elenco dei doni. } \\
\text { Conclusione: rispettare i compagni e scagliarsi } \\
\quad \underline{\text { contro i nemici. }}\end{array}$ & $\begin{array}{c}69 \\
68 \\
5\end{array}$ \\
\hline & $\begin{array}{c}\text { Ach. } \\
\text { 308-429 }\end{array}$ & $\begin{array}{l}\text { Odisseo non ha citato i torti subiti e le offese. } \\
\text { Agamennone è un codardo. } \\
\underline{\text { I doni sono odiosi e non valgono quanto l'amata }} \\
\underline{\text { sposa. }} \\
\underline{\text { Le imprese sono dovute solo ad Achille e non ad }} \\
\quad \underline{\text { Agamennone. }} \\
\underline{\text { Ironia sul motivo della guerra: Menelao non ha }} \\
\underline{\text { forse fatto la guerra ai Troiani per una donna? }}\end{array}$ & $\begin{array}{c}10 \\
13 \\
17 ; 18 ; 23 ; \\
27 ; 28 ; \text { ecc. } \\
49 \\
36-40\end{array}$ \\
\hline & $\begin{array}{c}\text { Fe. } \\
434-605\end{array}$ & 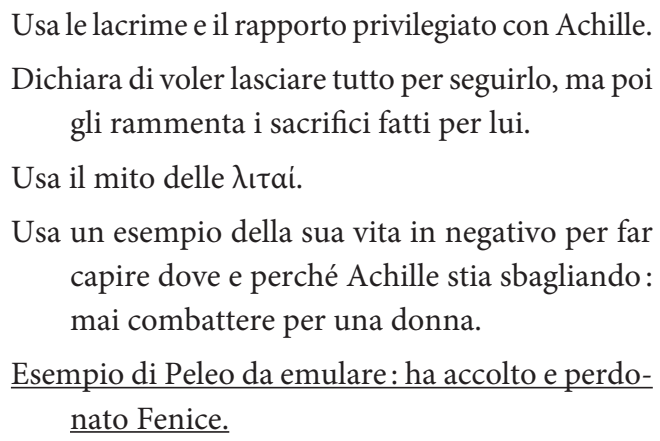 & 5 \\
\hline
\end{tabular}




\begin{tabular}{|c|c|c|c|}
\hline & $\begin{array}{c}\text { Ach. } \\
607-619\end{array}$ & $\begin{array}{l}\text { Risposta semplice, ma decisa: invece di piangere, } \\
\text { l'anziano amico dovrebbe schierarsi e scegliere } \\
\text { da che parte stare. } \\
\text { Il discorso di Fenice - basato sull'emotività del } \\
\text { rapporto personale e sulle lacrime - funziona } \\
\text { in parte. }\end{array}$ & $64 ; 67$ \\
\hline & $\begin{array}{c}\text { Ai. } \\
624-642\end{array}$ & $\begin{array}{l}\text { Parla ad Achille in terza persona. } \\
\text { Lascia, a ragione, Agamennone fuori dal discorso. } \\
\text { Esempio di assassino. } \\
\text { Ricordo del vincolo di amicizia e di ospitalità : } \\
\quad \text { lo spirito da camerata di Aiace deve funzionare. }\end{array}$ & 35 \\
\hline & $\begin{array}{c}\text { Ach. } \\
644-655\end{array}$ & $\begin{array}{l}\text { Achille conferma che Aiace ha parlato bene. } \\
\text { Ribadisce la gravità della sua offesa, che è stata } \\
\text { pubblica. }\end{array}$ & \\
\hline XI & $\begin{array}{c}\mathrm{Ne} . \\
656-803\end{array}$ & $\begin{array}{l}\text { Indifferenza di Achille per gli Achei. } \\
\text { Rimpianto per la sua giovinezza perduta. } \\
\text { (vv. 671-762) Lungo racconto biografico: esso è } \\
\text { paradigmatico per suffragare il monito che sta } \\
\text { per dare a Patroclo e ribadire la propria autorità. } \\
\text { Secondo racconto: parole di Menezio, utili a ram- } \\
\quad \text { mentare a Patroclo il suo dovere. } \\
\text { Convincere Patroclo a convincere. } \\
\text { Nestore propone una sostituzione: un compro- } \\
\text { messo. }\end{array}$ & $\begin{array}{c}29 \\
6\end{array}$ \\
\hline
\end{tabular}




\begin{tabular}{|c|c|c|c|}
\hline \multirow{3}{*}{ XVI } & $\begin{array}{l}\text { Ach. } \\
\text { 7-19 }\end{array}$ & $\begin{array}{l}\text { Similitudine con una bambinetta. } \\
\text { Il solo motivo di pianto potrebbe essere la morte } \\
\text { dei genitori. } \\
\text { Al massimo, un altro motivo potrebbe essere la } \\
\text { pietà per gli Achei. }\end{array}$ & 1 \\
\hline & $\begin{array}{c}\mathrm{Pa} . \\
21-45\end{array}$ & $\begin{array}{l}\text { Captatio benevolentiae } \\
\text { Brevissima descrizione della disperazione degli } \\
\text { Achei. } \\
\text { Auspicio di non provare mai un desiderio di rabbia } \\
\quad \text { così devastante. } \\
\text { Riferimento alla sua doppia ascendenza. } \\
\text { Accenno ad un misterioso oracolo di cui l'avrebbe } \\
\quad \text { informato la madre. } \\
\text { Esortazione a cedergli le armi e a mandarlo a } \\
\quad \text { condurre i Mirmidoni al suo posto. }\end{array}$ & 29 \\
\hline & $\begin{array}{c}\text { Ach. } \\
\text { 49-100 }\end{array}$ & $\begin{array}{l}\text { Proposta: lasciare stare il passato e andare avanti. } \\
\text { Acconsente a mandare Patroclo al suo posto. } \\
\text { Monito: Patroclo non deve privarlo della gloria. } \\
\text { Preghiera: possano gli dei vedere i due amici } \\
\quad \text { conquistare da soli la gloria. }\end{array}$ & $\begin{array}{l}10 ; 17 ; 18 ; \\
23 ; 27 ; 28 ; \\
\text { ecc. } \\
\quad 42\end{array}$ \\
\hline
\end{tabular}


